Proyecciones

Vol. 22, No 3, pp. 243-271, December 2003.

Universidad Católica del Norte

Antofagasta - Chile

\title{
SUR LE PROBLÈME ADDITIF DE COUSIN BASIQUE
}

\author{
AZIZ EL KACIMI \\ Universitè de Valenciennes, Francia \\ and \\ TOUSSAINT SOHOU \\ Université de Cocody, UFR de Mathématiques, Francia
}

\begin{abstract}
L'objet de ce travail est l'étude du problème additif de Cousin basique pour un feuilletage transversalement holomorphe. Plusieurs exemples ont été examinés et plus particulièrement les feuilletages obtenus par suspension d'un groupe de biholomorphismes d'une variété analytique complexe.

Pendant l'élaboration de ce travail, le second auteur a effectué une série de séjours à l'Université de Valenciennes au sein du LAMATH. Il remercie cette institution pour son hospitalité ainsi que le CIES qui a organisé ces séjours et le Ministère Français des Affaires Etrangères pour les avoir financés.
\end{abstract}




\section{Introduction}

Soient $M$ une variété analytique complexe et $\mathcal{U}=\left\{U_{i}\right\}$ un recouvrement ouvert localement fini de $M$; sur chaque intersection non vide $U_{i j}=U_{i} \cap U_{j}$ on se donne une fonction holomorphe $f_{i j}$ et on suppose que $f_{j i}=-f_{i j}$ et que, sur toute triple intersection $U_{i j k}=U_{i} \cap U_{j} \cap U_{k}$, la famille $\left\{f_{i j}\right\}$ vérifie la condition (dite de cocycle) $f_{j k}-f_{i k}+f_{i j}=0$. Pour chaque $i \in I$, existe$t$-il une fonction $f_{i}$ définie et holomorphe sur $U_{i}$ et telle que $f_{j}-f_{i}=f_{i j}$ sur $U_{i j}$ ? C'est le problème additif de Cousin pour le recouvrement $\left\{U_{i}\right\}$ et la donnée $\left\{f_{i j}\right\}$. Il a été posé et résolu par P. Cousin lui-même dans [Co] pour un polydisque de $\mathbf{C}^{n}$. Sur un ouvert $\Omega$ de $\mathbf{C}$, ce problème admet toujours une solution [Hö].

On voit, de façon évidente, que toute donnée de Cousin pour un recouvrement $\mathcal{U}=\left\{U_{i}\right\}$ de la variété complexe $M$ définit un 1-cocycle à valeurs dans le faisceau $\mathcal{O}$ des germes de fonctions holomorphes sur $M$; ce cocycle est un cobord si, et seulement si, le problème de Cousin pour cette donnée admet une solution. L'espace $H^{1}(\mathcal{U}, \mathcal{O})$ contient donc exactement les obstructions à la résolution d'un tel problème. Il n'est pas difficile de voir que cette théorie se généralise à toute orbifold complexe (i.e. un espace complexe localement modelé sur le quotient d'un ouvert de $\mathbf{C}^{n}$ par une action d'un groupe fini de biholomorphismes). On peut alors se demander comment peut-on approcher cette question pour une classe plus large d'espaces complexes, en l'occurrence l'espace des feuilles $M / \mathcal{F}$ d'un feuilletage $\mathcal{F}$ transversalement holomorphe sur une variété différentiable $M$. A la variété feuilletée $(M, \mathcal{F})$ est associé un faisceau naturel : le faisceau $\mathcal{O}_{\mathcal{F}}$ des germes de fonctions basiques holomorphes; il est pour l'espace des feuilles ce qu'est le faisceau $\mathcal{O}$ pour une variété complexe. L'objet de ce papier est d'enclencher le pas dans l'étude de ce qu'on appellera désormais le problème additif de Cousin basique. Le cas des feuilletages tangentiellement holomorphes a été examiné dans [Ek3] et [GT].

Dans la section 1 on rappelle les définitions de base sur les feuilletages transversalement holomorphes et les divers objets basiques qui leur sont rattachés. Dans la section 2 on définit le faisceau $\mathcal{O}_{\mathcal{F}}^{p}$ des germes de $p$-formes basiques holomorphes et on en donne une résolution fine et elliptique en termes de formes différentielles ; cette résolution se trouve dans [DK] mais elle a déjà été donnée par I. Vaisman dans [Va]. Dans la section 3 on énonce le problème additif de Cousin basique et on donne quelques méthodes générales de calcul, qu'on applique : dans la section 4 aux revêtements feuilletés et dans les sections $5,6,7$ et 8 à divers exemples de feuilletages obtenus par suspension d'un groupe $\Gamma$ de biholomorphismes d'une variété 
complexe $F$. On montre que le calcul de $H^{*}\left(M, \mathcal{O}_{\mathcal{F}}^{p}\right)$ se ramène souvent à celui de la cohomologie du groupe discret $\Gamma$ à valeurs dans le $\Gamma$-module $\mathcal{O}(F)$ des fonctions holomorphes sur $F$. Des calculs explicites sont faits et en particulier lorsque $\Gamma=\mathbf{Z}$.

Dans toute la suite, $M$ sera une variété différentiable (de classe $C^{\infty}$ ) de dimension $m+2 n$. On la supposera connexe et orientable. Tout recouvrement ouvert de $M$ que l'on considérera sera localement fini i.e. tout compact de $M$ ne rencontre qu'un nombre fini d'ouverts de ce recouvrement.

\section{Eléments basiques d'un feuilletage}

1.1. Définition. Un feuilletage transversalement holomorphe $\mathcal{F}$ de codimension (complexe) $n$ sur $M$ est donné par un recouvrement ouvert $\left\{U_{i}\right\}_{i \in I}$ et des difféomorphismes $\Omega_{i} \times \mathbf{R}^{m} \stackrel{\varphi_{i}}{\longrightarrow} U_{i}$ (où $\Omega_{i}$ est un ouvert de $\mathbf{C}^{n}$ ) tels que, sur $U_{i} \cap U_{j} \neq \emptyset$, le changement de coordonnées $\varphi_{j}^{-1} \circ \varphi_{i}(z, x)=\left(z^{\prime}, x^{\prime}\right)$ soit de la forme $z^{\prime}=\gamma_{i j}(z)$ et $x^{\prime}=\varphi_{i j}(z, x)$ avec $\gamma_{i j}$ holomorphe. On dira que $U_{i} \simeq \Omega_{i} \times \mathbf{R}^{m}$ est un ouvert distingué pour $\mathcal{F}$; il sera désormais identifié à $\Omega_{i} \times \mathbf{R}^{m}$.

Une telle structure peut être vue comme une partition de la variété $M$ en sous-variétés différentiables immergées, appelées feuilles de $\mathcal{F}$; cette partition étant indexée localement par un ouvert de $\mathbf{C}^{n}$ et le passage d'une feuille à une autre se faisant à l'aide d'une transformation biholomorphe. Elle peut aussi s'interpréter comme la famille des variétés intégrales d'un système différentiel dont les solutions dépendent holomorphiquement d'un paramètre complexe.

Soient $M$ et $M^{\prime}$ deux variétés munies respectivement de feuilletages transversalement holomorphes $\mathcal{F}$ et $\mathcal{F}^{\prime}$. Un morphisme de $(M, \mathcal{F})$ vers $\left(M^{\prime}, \mathcal{F}^{\prime}\right)$ est une application différentiable $\varphi: M \longrightarrow M^{\prime}$ telle que, pour tout ouvert $U$ de $M$ distingué pour $\mathcal{F}$ et tout ouvert $U^{\prime}$ de $M^{\prime}$ distingué pour $\mathcal{F}^{\prime}$ et tel que $V=\varphi(U) \cap U^{\prime}$ soit non vide, l'application $\varphi: U \longrightarrow V$ soit de la forme $\varphi(z, x)=(\gamma(z), \psi(z, x))$ avec $\gamma$ holomorphe. Si en plus $\varphi$ est un difféomorphisme, on dira que $\varphi$ est un isomorphisme de $(M, \mathcal{F})$ $\operatorname{sur}\left(M^{\prime}, \mathcal{F}^{\prime}\right)$. Dans ce cas les feuilletages $\mathcal{F}$ et $\mathcal{F}^{\prime}$ ont même dimension et même codimension. Si $M=M^{\prime}$ et $\mathcal{F}=\mathcal{F}^{\prime}$ un isomorphisme $\varphi:(M, \mathcal{F}) \longrightarrow$ $\left(M^{\prime}, \mathcal{F}^{\prime}\right)$ sera appelé automorphisme de $\mathcal{F}$. On dira que deux feuilletages $\mathcal{F}$ et $\mathcal{F}^{\prime}$ transversalement holomorphes sur $M$ sont conjugués s'il existe un isomorphisme $\varphi:(M, \mathcal{F}) \longrightarrow\left(M, \mathcal{F}^{\prime}\right)$. 


\subsection{Exemples}

(1) Une variété analytique complexe $M$ de dimension $n$ donne lieu, de façon naturelle, à un feuilletage transversalement holomorphe de codimension $n$ : les feuilles sont les points de $M$.

(2) Tout feuilletage holomorphe au sens usuel sur une variété analytique complexe est un feuilletage transversalement holomorphe sur la variété réelle sous-jacente.

(3) Soient $F$ une variété complexe de dimension $n$ et $B$ une variété différentiable de dimension $m$. On note $\Gamma=\pi_{1}(B)$ le groupe fondamental de $B$ et on suppose qu'il existe une représentation $\rho$ de $\Gamma$ dans le groupe $\operatorname{Aut}(F)$ des biholomorphismes de $F$. Le feuilletage $\widetilde{\mathcal{F}}$ sur $\widetilde{M}=F \times \widetilde{B}(\widetilde{B}$ étant le revêtement universel de $B$ ) dont les feuilles sont $\{z\} \times \widetilde{B}$ avec $z \in F$, est transversalement holomorphe et invariant par toutes les transformations $\Phi: F \times \widetilde{B} \longrightarrow F \times \widetilde{B}, \Phi(z, x)=(\rho(\gamma)(z), \gamma x)$ où $\gamma \in \Gamma$ et $\gamma x$ est l'action de $\gamma$ sur $x$ ( $\gamma$ est un automorphisme du revêtement $\widetilde{B} \longrightarrow B$ ); $\widetilde{\mathcal{F}}$ induit donc un feuilletage transversalement holomorphe $\mathcal{F}$ sur la variété quotient $M=$ $F \times \widetilde{B} /(z, x) \simeq(\rho(\gamma)(z), \gamma x) ;$ il est transverse à la fibration $F \longrightarrow M \stackrel{\pi}{\longrightarrow} B$ où $\pi$ est induite par la deuxième projection $(z, x) \in F \times \widetilde{B} \longmapsto x \in \widetilde{B}$. On dira que $\mathcal{F}$ est le feuilletage obtenu par suspension de la représentation $\rho$.

(4) Soit $\widetilde{M}$ une variété de dimension $m+2 n$ munie d'un feuilletage transversalement holomorphe $\widetilde{\mathcal{F}}$ de codimension $n$. Soit $\Gamma$ un groupe (dénombrable) discret agissant sur $\widetilde{M}$ de façon libre et propre par automorphismes de $\widetilde{\mathcal{F}}$. Alors le feuilletage $\mathcal{F}$, induit sur la variété quotient $M=\widetilde{M} / \Gamma$, est transversalement holomorphe de codimension $n$. Un tel exemple de feuilletage sera appelé revêtement feuilleté. On retrouve une suspension si on prend pour $\widetilde{\mathcal{F}}$ le feuilletage dont les feuilles sont les fibres de la première projection $\widetilde{M}=F \times \widetilde{B} \longrightarrow F$ (où $\widetilde{B}$ est une variété différentiable de dimension $m$ simplement connexe et $F$ une variété complexe de dimension $n$ ) et $\Gamma$ agissant librement proprement sur $\widetilde{B}$ et par biholomorphismes sur $F$.

(5) On pose $\widetilde{M}=\mathbf{C}^{n} \times \mathbf{R}^{m} \backslash\{(0,0)\}$ et on note $\widetilde{\mathcal{F}}$ le feuilletage défini par l'équation $d z=0$ où $(z, x)$ sont les coordonnées d'un point de $\widetilde{M}$. Ce feuilletage est invariant par la transformation

$$
(z, x) \in \widetilde{M} \longmapsto(\lambda z, \lambda x) \in \widetilde{M}
$$

(où $\lambda \in] 0,1[$ ) ; il induit donc un feuilletage $\mathcal{F}$ sur la variété de Hopf 


$$
M=\widetilde{M} /(z, x) \simeq(\lambda z, \lambda x)
$$

qui est difféomorphe à $\mathbf{S}^{1} \times \mathbf{S}^{N}$ avec $N=m+2 n-1$; les feuilles sont des plans $\mathbf{R}^{m}$ sauf celle correspondant à $z=0$ qui est difféomorphe à $\mathbf{S}^{1} \times \mathbf{S}^{m-1}$. Ceci est un exemple concret de la situation (4).

\subsection{Eléments basiques}

Soit $\mathcal{F}$ un feuilletage transversalement holomorphe de dimension (réelle) $m$ et de codimension (complexe) $n$ sur $M$. On notera $T \mathcal{F}$ le fibré tangent à $\mathcal{F}$ qui est un sous-fibré du fibré tangent $T M$ à $M$ et $\nu \mathcal{F}=T M / T \mathcal{F}$ le fibré normal. L'espace $\chi(\mathcal{F})$ des sections globales de $T \mathcal{F}$ (champs tangents à $\mathcal{F}$ ) est un module sur l'algèbre $A(M)$ des fonctions de classe $C^{\infty}$ sur $M$.

Une forme différentielle $\alpha$ sur $M$ est dite basique, si elle vérifie $i_{X} \alpha=0$ et $L_{X} \alpha=0$ pour tout $X \in \chi(\mathcal{F})\left(i_{X}\right.$ et $L_{X}$ désignent respectivement le produit intérieur et la dérivée de Lie associés au champ $X$ ). Une telle forme est localement l'image réciproque d'une forme sur $\mathbf{C}^{n}$. Une fonction basique est une fonction constante sur les feuilles de $\mathcal{F}$. Il est immédiat de voir que si la forme $\alpha$ est basique, il en est de même pour $d \alpha$; ainsi les formes basiques constituent un sous-complexe $\left(A^{*}(M / \mathcal{F}), d\right)$ du complexe de de Rham de $M$. Son homologie $H^{*}(M / \mathcal{F})$ s'appelle la cohomologie basique de $\mathcal{F}$. Elle joue le rôle de la cohomologie de de Rham pour l'espace des feuilles $B=M / \mathcal{F}$ (cf. [Ek2] pour les détails).

En usant de la structure complexe transverse à $\mathcal{F}$ on peut, pour tout entier $r \in\{0, \ldots, 2 n\}$, décomposer l'espace vectoriel $A^{r}(M / \mathcal{F})$ sous forme d'une somme directe

$$
A^{r}(M / \mathcal{F})=\bigoplus_{p+q=r} A^{p q}(M / \mathcal{F})
$$

Un élément de $A^{p q}(M / \mathcal{F})$ est appelé forme basique de type $(p, q)$. Localement, une telle forme s'écrit

$$
\alpha=\sum_{i_{1} \cdots i_{p} j_{1} \cdots j_{q}} \alpha_{i_{1} \cdots i_{p} j_{1} \cdots j_{q}} d z_{i_{1}} \wedge \cdots d z_{i_{p}} \wedge d \bar{z}_{j_{1}} \wedge \cdots \wedge d \bar{z}_{j_{q}}
$$

où les $\alpha_{i_{1} \cdots i_{p} j_{1} \cdots j_{q}}$ sont des fonctions $C^{\infty}$ qui ne dépendent que de $z$. La restriction de $d$ à $A^{r}(M / \mathcal{F})$ se décompose, comme dans le cas classique, en une somme $d=\partial+\bar{\partial}$ où $\partial$ et $\bar{\partial}$ sont deux opérateurs respectivement de types $(1,0)$ et $(0,1)$. Si on fixe $p$, on obtient un complexe différentiel

$$
0 \longrightarrow A^{p 0}(M / \mathcal{F}) \stackrel{\bar{\partial}}{\longrightarrow} A^{p 1}(M / \mathcal{F}) \stackrel{\bar{\partial}}{\longrightarrow} \cdots \stackrel{\bar{\partial}}{\longrightarrow} A^{p n}(M / \mathcal{F}) \longrightarrow 0
$$


appelé complexe basique de Dolbeault dont l'homologie sera notée $H^{p *}(M / \mathcal{F})$ et appelée cohomologie basique de Dolbeault de $\mathcal{F}$.

Une $p$-forme basique holomorphe est une forme basique $\omega$ de type $(p, 0)$ et vérifiant $\bar{\partial} \omega=0$. On notera $\mathcal{O}_{\mathcal{F}}^{p}$ le faisceau des germes de telles formes ; pour $p=0$ on conviendra de noter $\mathcal{O}_{\mathcal{F}}$ le faisceau $\mathcal{O}_{\mathcal{F}}^{0}$ qui sera donc le faisceau des germes de fonctions basiques holomorphes ; lorsque la dimension de $\mathcal{F}$ est $m=0$ i.e. $M$ est une variété analytique complexe, $\mathcal{O}_{\mathcal{F}}$ se réduit au faisceau $\mathcal{O}$ des germes de fonctions holomorphes. Ces faisceaux ne sont pas fins et donnent lieu à une théorie de cohomologie $H^{*}\left(M, \mathcal{O}_{\mathcal{F}}^{p}\right)$, en général non triviale.

On peut remarquer que si $\mathcal{F}$ et $\mathcal{F}^{\prime}$ sont deux feuilletages transversalement holomorphes conjugués alors le faisceau $\mathcal{O}_{\mathcal{F}^{\prime}}$ est isomorphe au faisceau $\mathcal{O}_{\mathcal{F}}$ et les cohomologies $H^{*}\left(M / \mathcal{F}^{\prime}\right)$ et $H^{p *}\left(M / \mathcal{F}^{\prime}\right)$ sont isomorphes respectivement à $H^{*}(M / \mathcal{F})$ et $H^{p *}(M / \mathcal{F})$.

\section{Résolution du faisceau $\mathcal{O}_{\mathcal{F}}^{p}$}

Nous la donnerons en suivant la démarche de [DK]. Soit $U$ un ouvert de $M$ distingué pour $\mathcal{F}$; on le munit du feuilletage induit qu'on notera toujours $\mathcal{F}$. Par définition même de $U, \mathcal{F}$ admet un feuilletage transverse $\mathcal{V}$ dont les feuilles sont des variétés analytiques complexes de dimension $n$.

Le fibré tangent à $U$ se décompose en une somme $T U=T \mathcal{V} \oplus T \mathcal{F}$ où $T \mathcal{V}$ et $T \mathcal{F}$ sont les fibrés tangents respectivement à $\mathcal{V}$ et $\mathcal{F}$. Comme $T \mathcal{V}$ a une structure complexe, le complexifié $T U \otimes \mathbf{C}$ se décompose de la façon suivante

$T U \otimes \mathbf{C}=\nu^{10} \oplus \nu^{01} \oplus(T \mathcal{F} \otimes \mathbf{C})$ où $\nu^{10}$ est la partie holomorphe de $T \mathcal{V} \otimes \mathbf{C}$ et $\nu^{01}$ sa partie anti-holomorphe. Ceci permet de mettre une trigraduation sur l'espace $A^{r}(U)$ des $r$-formes différentielles sur $U$

$$
A^{r}(U)=\bigoplus_{p+k+\ell=r} A^{p k \ell}(U) .
$$

Un élément de $A^{p k \ell}(U)$ est appelé forme différentielle de type $(p, k, \ell)$. La différentielle $d: A^{r}(U) \longrightarrow A^{r+1}(U)$ se décompose en la somme de trois opérateurs $d=\partial+\bar{\partial}+d_{\mathcal{F}} ; d_{\mathcal{F}}$ est la différentielle extérieure le long des feuilles de $\mathcal{F}$ et est de type $(0,0,1) ; \bar{\partial}$ est l'opérateur de Cauchy-Riemann le long des feuilles de $\mathcal{V}$ et est de type $(0,1,0) ; \partial$ est de type $(1,0,0)$. Un calcul immédiat, utilisant le fait que $d^{2}=0$, montre que

$$
d_{\mathcal{F}}^{2}=0, \quad \bar{\partial}^{2}=0 \text { et } d_{\mathcal{F}} \bar{\partial}+\bar{\partial} d_{\mathcal{F}}=0 .
$$


On en déduit alors $\left(d_{\mathcal{F}}+\bar{\partial}\right)^{2}=0$. Il est facile de voir que les fonctions basiques holomorphes sur $U$ sont exactement les fonctions $f$ sur $U$ qui vérifient $d_{\mathcal{F}} f=0$ et $\bar{\partial} f=0$.

Notons $\mathcal{A}^{\text {pkl }}$ le faisceau des germes de formes différentielles de type $(p, k, \ell)$. Alors $\mathcal{O}_{\mathcal{F}}^{p}$ est le noyau de l'opérateur

$$
D:=\bar{\partial}+d_{\mathcal{F}}: \mathcal{A}^{p 00} \longrightarrow \mathcal{A}^{p 10} \oplus \mathcal{A}^{p 01} .
$$

On fixe $p$ et on pose

$$
A^{q}(U)=\bigoplus_{k+\ell=q} A^{p k \ell}(U) \text { et } \mathcal{A}^{q}=\bigoplus_{k+\ell=q} \mathcal{A}^{p k \ell} .
$$

On dira que $\alpha$ est $D$-fermée si $D \alpha=0, D$-exacte s'il existe $\beta$ telle que $\alpha=D \beta$. Tout élément $\alpha$ de $A^{q}(U)$ s'écrit

$$
\alpha=\sum_{k+\ell=q} \alpha_{(k, \ell)}
$$

avec $\alpha_{(k, \ell)} \in A^{p k \ell}$. Le lemme suivant est un ingrédient essentiel pour la résolution du faisceau $\mathcal{O}_{\mathcal{F}}^{p}$. C'est un "mélange" du lemme de Poincaré et du lemme de Dolbeault.

2.1. Lemme de Poincaré-Dolbeault. Soit $\alpha=\sum \alpha_{(k, \ell)} \in A^{q}(U)$ une forme $D$-fermée. Alors, pour tout point y de $U$, il existe un voisinage ouvert $V$ de $y$ et une forme $\beta \in A^{q-1}(V)$ telle que $\alpha=D \beta$ sur $V$.

DÉmonstration. L'équation $D \alpha=0$ est équivalente au système

$$
\left\{\begin{array}{ccc}
d_{\mathcal{F}} \alpha_{(0, q)} & = & 0 \\
d_{\mathcal{F}} \alpha_{(1, q-1)} & = & -\bar{\partial} \alpha_{(0, q)} \\
d_{\mathcal{F}} \alpha_{(2, q-2)} & = & -\bar{\partial} \alpha_{(1, q-1)} \\
\cdots & = & \cdots \\
d_{\mathcal{F}} \alpha_{(q, 0)} & = & -\bar{\partial} \alpha_{(q-1,1)} \\
0 & = & \bar{\partial} \alpha_{(q, 0)}
\end{array}\right.
$$

Soit $y$ un point de $U$; nous allons construire de proche en proche l'ouvert $V$ et la forme $\beta$ en utilisant les différentes équations de ce système.

i) La forme $\alpha_{(0, q)}$ est $d_{\mathcal{F}}$-fermée ; par le lemme de Poincaré à paramètre, il existe un ouvert $U_{0} \subset U$ contenant $y$ et une forme $\beta_{(0, q-1)}$ définie sur $U_{0}$ telle que $d_{\mathcal{F}} \beta_{(0, q-1)}=\alpha_{(0, q)}$.

ii) Sur $U_{0}$, on a $\bar{\partial} \alpha_{(0, q)}=\bar{\partial} d_{\mathcal{F}} \beta_{(0, q-1)}=-d_{\mathcal{F}} \bar{\partial} \beta_{(0, q-1)}$. La deuxième équation du système donne alors $d_{\mathcal{F}} \alpha_{(1, q-1)}=d_{\mathcal{F}} \bar{\partial} \beta_{(0, q-1)}$ et donc

$$
\alpha_{(1, q-1)}=\bar{\partial} \beta_{(0, q-1)}+\eta_{(1, q-1)}
$$


avec $d_{\mathcal{F}} \eta_{(1, q-1)}=0$; comme $\eta_{(1, q-1)}$ est $d_{\mathcal{F}}$-fermée sur $U_{0}$, il existe un ouvert $U_{1} \subset U_{0}$ et une forme $\beta_{(1, q-2)}$ définie sur $U_{1}$ telle que $d_{\mathcal{F}} \beta_{(1, q-2)}=\eta_{(1, q-1)}$. Finalement on a, sur $U_{1}$,

$$
\alpha_{(1, q-1)}=\bar{\partial} \beta_{(0, q-1)}+d_{\mathcal{F}} \beta_{(1, q-2)} .
$$

iii) Calculons $\bar{\partial} \alpha_{(1, q-1)}$ dans l'égalité précédente ; on obtient

$$
\bar{\partial} \alpha_{(1, q-1)}=\bar{\partial} d_{\mathcal{F}} \beta_{(1, q-2)}=-d_{\mathcal{F}} \bar{\partial} \beta_{(1, q-2)}
$$

et d'après la troisième équation du système

$$
d_{\mathcal{F}} \alpha_{(2, q-2)}=d_{\mathcal{F}} \bar{\partial} \beta_{(1, q-2)} .
$$

Ce qui donne

$$
\alpha_{(2, q-2)}=\bar{\partial} \beta_{(1, q-2)}+\eta_{(2, q-2)}
$$

avec $d_{\mathcal{F}} \eta_{(2, q-2)}=0$; il existe alors un ouvert $U_{2} \subset U_{1}$ et une forme $\beta_{(2, q-3)}$ définie sur $U_{2}$ telle que $d_{\mathcal{F}} \beta_{(2, q-3)}=\eta_{(2, q-2)}$; d'où

$$
\alpha_{(2, q-2)}=\bar{\partial} \beta_{(1, q-2)}+d_{\mathcal{F}} \beta_{(2, q-3)} .
$$

iv) De cette manière on construit une suite finie décroissante d'ouverts $U_{q-1} \subset U_{q-2} \subset \cdots \subset U_{1} \subset U_{0}$ et une suite finie de formes différentielles $\beta_{(0, q-1)}, \cdots, \beta_{(q-1,0)}$ avec $\beta_{(i, q-1-i)}$ définie sur $U_{i}$ vérifiant les conditions suivantes

$$
\left\{\begin{array}{ccc}
\alpha_{(0, q)} & = & d_{\mathcal{F}} \beta_{(0, q-1)} \\
\alpha_{(1, q-1)} & = & \bar{\partial} \beta_{(0, q-1)}+d_{\mathcal{F}} \beta_{(1, q-2)} \\
\cdots & = & \cdots \\
\alpha_{(q-1,1)} & = & \bar{\partial} \beta_{(q-2,1)}+d_{\mathcal{F}} \beta_{(q-1,0)} \\
\alpha_{(q, 0)} & = & \bar{\partial} \beta_{(q-1,0)}
\end{array}\right.
$$

v) Il n'est pas difficile de voir que $\beta=\sum \beta_{\left(k^{\prime}, \ell^{\prime}\right)}$ (où la sommation porte sur tous les $k^{\prime}$ et $\ell^{\prime}$ tels que $k^{\prime}+\ell^{\prime}=q-1$ ) est bien définie sur $V=U_{q-1}$ et qu'elle vérifie $D \beta=\alpha$; c'est la forme différentielle cherchée.

2.2. Lemme d'ellipticité. Soit $U$ un ouvert distingué pour le feuilletage; alors le complexe différentiel $0 \longrightarrow A^{0}(U) \stackrel{D}{\longrightarrow} A^{1}(U) \stackrel{D}{\longrightarrow} \cdots \stackrel{D}{\longrightarrow}$ $A^{m+n}(U) \longrightarrow 0$ est elliptique.

DÉmonstration. Pour tout $y \in U$, notons $\Lambda_{y}^{q}$ la fibre au point $y$ du fibré vectoriel

$$
\Lambda^{q}=\bigoplus_{k+\ell=q} \Lambda^{p, k, \ell}
$$


Soit $\xi=\xi_{\nu}^{+}+\xi_{\nu}^{-}+\xi_{\mathcal{F}}$ un covecteur tangent à $U$ en $y$. Le symbole de l'opérateur différentiel du premier ordre $A^{q}(U) \stackrel{D}{\longrightarrow} A^{q+1}(U)$ est l'application linéaire $\sigma(D)(y, \xi): \Lambda_{y}^{q} \longrightarrow \Lambda_{y}^{q+1}$ définie par

$$
\sigma(D)(y, \xi)(v)=\xi_{\nu}^{-} \wedge v+\xi_{\mathcal{F}} \wedge v .
$$

Comme $\xi=0$ si, et seulement si, $\xi_{\nu}^{-}=0$ et $\xi_{\mathcal{F}}=0$, la suite des symboles

$$
\cdots \longrightarrow \Lambda_{y}^{q} \stackrel{\sigma(D)(y, \xi)}{\longrightarrow} \Lambda_{y}^{q+1} \longrightarrow \cdots
$$

est exacte pour tout $\xi$ non nul ; ce qui établit l'ellipticité du complexe.

Nous allons maintenant étudier la situation globale sur $M$. Si $E \longrightarrow M$ est un fibré vectoriel, on conviendra de noter $A(E)$ l'espace de ses sections de classe $C^{\infty}$ et $\mathcal{A}(E)$ le faisceau des germes de sections associé. Le complexifié $\nu \mathrm{du}$ fibré normal $\nu \mathcal{F}=T M / T \mathcal{F}$ s'écrit sous la forme $\nu=\nu^{10} \oplus \nu^{01}$. On a une suite exacte

$$
0 \longrightarrow T \mathcal{F} \otimes \mathbf{C} \hookrightarrow T M \otimes \mathbf{C} \stackrel{\tau}{\longrightarrow} \nu^{10} \oplus \nu^{01} \longrightarrow 0 .
$$

Notons $\tau_{1}$ l'application $p_{1} \circ \tau$ où $p_{1}$ est la première projection $\nu^{10} \oplus \nu^{01} \longrightarrow$ $\nu^{10}$. Soit $\zeta$ le noyau de $\tau_{1}$. On obtient alors une suite exacte

$$
0 \longrightarrow \zeta \stackrel{j}{\hookrightarrow} T M \otimes \mathbf{C} \stackrel{\tau_{1}}{\longrightarrow} \nu^{10} \longrightarrow 0 .
$$

En utilisant une section $\nu^{10} \longrightarrow T M \otimes \mathbf{C}$ de $\tau_{1}$, on construit des isomorphismes (non canoniques) de fibrés

$$
T M \otimes \mathbf{C} \simeq \nu^{10} \oplus \zeta \quad \text { et } \quad \zeta \simeq \nu^{01} \oplus(T \mathcal{F} \otimes \mathbf{C}) .
$$

Soit $\theta: T M \otimes \mathbf{C} \longrightarrow \zeta$ la deuxième projection et, pour tout entier naturel $\ell$, posons $\Sigma^{\ell}=\Lambda^{1}\left(\nu^{10}\right)^{*} \wedge \Lambda^{\ell-1} T^{*} M \otimes \mathbf{C}$. La suite (2) donne une suite exacte de fibrés

$$
0 \longrightarrow \Sigma^{r} \longrightarrow \Lambda^{r} T^{*} M \otimes \mathbf{C} \stackrel{j^{*}}{\longrightarrow} \Lambda^{r} \zeta^{*} \longrightarrow 0
$$

et une suite exacte au niveau des faisceaux des germes de sections associés

$$
\underline{0 \longrightarrow \mathcal{A}\left(\Sigma^{r}\right) \longrightarrow \mathcal{A}\left(\Lambda^{r} T^{*} M \otimes \mathbf{C}\right) \stackrel{j^{*}}{\longrightarrow} \mathcal{A}\left(\Lambda^{r} \zeta^{*}\right) \longrightarrow 0}
$$

Comme le fibré $\zeta$ est intégrable, la différentielle extérieure $d$ induit un opérateur

$$
\widetilde{d}: \mathcal{A}\left(\Sigma^{r}\right) \longrightarrow \mathcal{A}\left(\Sigma^{r+1}\right) \text {. }
$$


Soit alors $\widetilde{D}$ l'unique opérateur différentiel d'ordre 1 qui fait commuter le diagramme

$$
\begin{array}{cccc}
0 \rightarrow & \mathcal{A}\left(\sum^{r}\right) \rightarrow & \mathcal{A}\left(\Lambda^{r} T^{*} M \otimes \mathbf{C}\right) \stackrel{j^{*}}{\rightarrow} & \mathcal{A}\left(\Lambda^{r} \zeta^{*}\right) \rightarrow 0 \\
& \downarrow \downarrow d & \downarrow d & \downarrow \widetilde{D} \\
& \rightarrow \mathcal{A}\left(\sum^{r+1}\right) \rightarrow & \mathcal{A}\left(\Lambda^{r+1} T^{*} M \otimes \mathbf{C}\right) \stackrel{j^{*}}{\rightarrow} & \mathcal{A}\left(\Lambda^{r+1} \zeta^{*}\right) \rightarrow 0
\end{array}
$$

On posera, pour simplifier, $A^{r}(M)=A\left(\Lambda^{r} \zeta^{*}\right)$ et on notera $\mathcal{A}^{r}$ le faisceau associé. L'opérateur $\widetilde{D}$ induit alors un opérateur

$$
D: A^{r}(M) \longrightarrow A^{r+1}(M)
$$

de carré nul. Sur un ouvert distingué $U$, l'espace $A^{r}(U)$ coïncide avec l'espace défini prédemment et l'opérateur $D$ n'est rien d'autre que $d_{\mathcal{F}}+\bar{\partial}$. En utilisant les lemmes 2.1 et 2.2 on montre alors le

2.3. Théorème. La suite $0 \longrightarrow \mathcal{O}_{\mathcal{F}}^{p} \hookrightarrow \mathcal{A}^{0} \stackrel{D}{\longrightarrow} \mathcal{A}^{1} \stackrel{D}{\longrightarrow} \ldots \stackrel{D}{\longrightarrow} \mathcal{A}^{m+n} \longrightarrow$ 0 est une résolution fine et elliptique du faisceau $\mathcal{O}_{\mathcal{F}}^{p}$. La cohomologie $H^{*}\left(M, \mathcal{O}_{\mathcal{F}}^{p}\right)$ de $M$ à valeurs dans le faisceau $\mathcal{O}_{\mathcal{F}}^{p}$ est donc isomorphe $\grave{a}$ l'homologie $H^{*}\left(A^{*}(M)\right)$ du complexe différentiel

$$
0 \longrightarrow A^{0}(M) \stackrel{D}{\longrightarrow} A^{1}(M) \stackrel{D}{\longrightarrow} \cdots \stackrel{D}{\longrightarrow} A^{m+n}(M) \longrightarrow 0
$$

Si $M$ est compacte, les espaces vectoriels $H^{*}\left(M, \mathcal{O}_{\mathcal{F}}^{p}\right)$ sont de dimension finie.

Si $n=0$, le feuilletage est constitué d'une seule feuille, la variété ellemême ; le théorème 2.2 n'est alors rien d'autre que le théorème de de Rham. Si $m=0, \mathcal{F}$ est le feuilletage par points, $M$ est une variété complexe de dimension $n$ et le théorème 2.2 se réduit au théorème de Dolbeault.

On peut montrer aussi que la cohomologie $H^{q}\left(M, \mathcal{O}_{\mathcal{F}}^{p}\right)$ vérifie la dualité de Serre i.e. pour tout $p \in\{0, \cdots, n\}$ et tout $q \in\{0, \cdots, n+m\}$, l'espace vectoriel $H^{q}\left(M, \mathcal{O}_{\mathcal{F}}^{p}\right)$ est canoniquement isomorphe à $H^{m+n-q}\left(M, \mathcal{O}_{\mathcal{F}}^{n-p}\right)$ (cf. [Ek1]).

\section{Le problème additif de Cousin basique}

Soit $\mathcal{F}$ un feuilletage transversalement holomorphe de codimension $n$ sur $M$. La restriction de $\mathcal{F}$ à tout ouvert $U$ sera encore notée $\mathcal{F}$; si $\left(i_{0}, \cdots, i_{s}\right)$ est un multi-indice d'éléments de $I, U_{i_{0} \cdots i_{s}}$ sera l'intersection $U_{i_{0}} \cap \cdots \cap U_{i_{s}}$. Pour tout ouvert $U$, on désigne par $\mathcal{O}(U / \mathcal{F})$ l'espace des fonctions basiques 
holomorphes sur $U$; en coordonnées locales $(z, x)$, un élément de cet espace est une fonction indépendante de $x$ et holomorphe en $z$.

3.1. Enoncé. Le problème additif de Cousin basique relativement au recouvrement $\mathcal{U}$ consiste en ceci : sur chaque $U_{i j}$ on se donne une fonction basique holomorphe $f_{i j}$ telle que $f_{i j}+f_{j i}=0$ et $f_{j k}-f_{i k}+f_{i j}=0$ sur $U_{i j k}$. Sur chaque $U_{i}$, on cherche une fonction basique holomorphe $f_{i}$ telle que, sur $U_{i j}$ on ait $f_{i j}=f_{j}-f_{i}$.

La famille $\left\{f_{i j}\right\}$ définit un 1-cocycle sur $\mathcal{U}$ à valeurs dans $\mathcal{O}_{\mathcal{F}}$. Le problème a une solution si ce cocycle est un cobord i.e. si sa classe de cohomologie dans $H^{1}\left(\mathcal{U}, \mathcal{O}_{\mathcal{F}}\right)$ est nulle. Par suite, le problème additif de Cousin basique pour $\mathcal{U}$ a une solution pour tout 1-cocycle $\left\{f_{i j}\right\}$ si, et seulement si, $H^{1}\left(\mathcal{U}, \mathcal{O}_{\mathcal{F}}\right)=0$. L'espace vectoriel $H^{1}\left(\mathcal{U}, \mathcal{O}_{\mathcal{F}}\right)$ contient donc exactement les obstructions à la résolution d'un tel problème.

Soit $q \in \mathbf{N}^{*}$. Un ouvert $U$ de $M$ (muni du feuilletage induit) est dit $q$ acyclique si $H^{q}\left(U, \mathcal{O}_{\mathcal{F}}\right)=0$; on dira que $U$ est acyclique s'il est $q$-acyclique pour tout $q \geq 1$. Un recouvrement ouvert $\mathcal{U}=\left\{U_{i}\right\}$ est dit acyclique si, pour tout multi-indice $\left(i_{0}, \cdots, i_{s}\right)$ de $I$, l'ouvert $U_{i_{0} \cdots i_{s}}$ est acyclique. L'espace $H^{0}\left(U, \mathcal{O}_{\mathcal{F}}\right)$ est constitué des fonctions basiques holomorphes (globales si $U=M)$ sur $U$. On le notera $\mathcal{O}_{\mathcal{F}}(U / \mathcal{F})$ et simplement $\mathcal{O}(U)$, si $\mathcal{F}$ est le feuilletage par points ; on munira ces espaces de la topologie de la convergence uniforme sur les compacts qui en fait des espaces de Fréchet.

\subsection{Exemples}

i) On considère la variété $M=\mathbf{C}^{2}-\{(0,0)\}$ munie du feuilletage holomorphe (et donc transversalement holomorphe) $\mathcal{F}$ dont les feuilles sont les courbes complexes d'équation $d z_{1}=0$; pour $z_{1} \neq 0$ les feuilles sont des droites affines complexes et celle correspondant à $z_{1}=0$ est le plan complexe épointé $\mathbf{C}^{*}$. Considérons le recouvrement ouvert $\mathcal{U}=\left\{U_{1}, U_{2}\right\}$ où

$$
\begin{gathered}
U_{1}=\left\{\left(z_{1}, z_{2}\right) \in M: z_{1} \neq 0\right\} \simeq \mathbf{C}^{*} \times \mathbf{C}, U_{2}=\left\{\left(z_{1}, z_{2}\right) \in M: z_{2} \neq 0\right\} \simeq \mathbf{C} \times \mathbf{C}^{*} \\
U_{1} \cap U_{2}=\mathbf{C}^{*} \times \mathbf{C}^{*} .
\end{gathered}
$$

Ce recouvrement n'est pas acyclique, donc ne calcule pas a priori le $H^{1}\left(M, \mathcal{O}_{\mathcal{F}}\right)$. On peut remarquer immédiatement que les fonctions basiques holomorphes sur $U_{1} \cap U_{2}$ sont exactement les fonctions basiques holomorphes sur $U_{1}$. Soit $f_{12}$ une fonction basique holomorphe sur $U_{1} \cap U_{2}$; on cherche $f_{1}$ basique holomorphe sur $U_{1}$ et $f_{2}$ basique holomorphe sur $U_{2}$ telles que $f_{12}=f_{2}-f_{1}$ sur $U_{1} \cap U_{2}$. On voit alors que les fonctions $f_{1}=-f_{12}$ et $f_{2}=0$ conviennent ; donc $H^{1}\left(\mathcal{U}, \mathcal{O}_{\mathcal{F}}\right)=0$ i.e. le problème additif de Cousin basique pour $\mathcal{U}$ 
a une solution. Mais le problème classique (celui qui correspond au feuilletage par points) n'en a pas ; en fait l'espace $H^{1}(\mathcal{U}, \mathcal{O})$ est de dimension infinie engendré par la famille ( $c f .[\mathrm{MK}]$ pour des calculs détaillés)

$$
\left\{\frac{1}{z_{1}^{n_{1}} z_{2}^{n_{2}}}: n_{1}, n_{2} \in \mathbf{N}\right\}
$$

ii) L'exemple qui suit est presque le précédent. On prend $M=\mathbf{C} \times \mathbf{R}$ privé du point $(0,0)$ et on le munit du feuilletage transversalement holomorphe $\mathcal{F}$ défini par $d z=0$. A l'aide du recouvrement ouvert acyclique $\mathcal{U}=\left\{U_{1}, U_{2}\right\}$ où

$U_{1}=\{(z, x) \in M: z \neq 0\} \simeq \mathbf{C}^{*} \times \mathbf{R}, U_{2}=\{(z, x) \in M: x \neq 0\} \simeq \mathbf{C} \times \mathbf{R}^{*}$

on montre que $H^{1}\left(\mathcal{U}, \mathcal{O}_{\mathcal{F}}\right)=H^{1}\left(M, \mathcal{O}_{\mathcal{F}}\right)=0$. On utilisera ce résultat par la suite pour calculer $H^{1}\left(\mathbf{S}^{2} \times \mathbf{S}^{1}, \mathcal{O}_{\mathcal{F}}\right)$ où la variété $\mathbf{S}^{2} \times \mathbf{S}^{1}$ est munie du feuilletage de l'exemple 1.2. (5).

3.3. Définition. On dira que $(M, \mathcal{F})$ est un feuilletage de Cousin si, pour tout recouvrement acyclique $\mathcal{U}$, le problème additif de Cousin basique relativement à $\mathcal{U}$ admet une solution $\left\{f_{i}\right\}$ pour toute donnée $\left\{f_{i j}\right\}$ i.e. si $H^{1}\left(\mathcal{U}, \mathcal{O}_{\mathcal{F}}\right)=0$. Si $\mathcal{F}$ est le feuilletage par points sur $M$ complexe, on a la notion de variété de Cousin.

La propriété pour un feuilletage $\mathcal{F}$ d'être de Cousin ne dépend que de sa classe de conjugaison en tant que feuilletage transversalement holomorphe.

\subsection{Remarque}

Le problème additif de Cousin basique pour $\mathcal{U}$ ne peut pas toujours s'interpréter comme le problème de Cousin classique sur l'espace des feuilles même dans le cas où celui-ci possède une structure de variété analytique complexe ( $c f$. le calcul fait dans la section 5.2).

\subsection{Le complexe de Cěch-de Rham-Dolbeault}

Nous allons d'abord montrer en suivant la démarche exposée dans [BT] que, pour un feuilletage transversalement holomorphe quelconque $(M, \mathcal{F})$, la cohomologie $H^{*}\left(\mathcal{U}, \mathcal{O}_{\mathcal{F}}\right)$ peut se calculer à l'aide d'un procédé du type Mayer-Vietoris. Nous ferons ensuite des calculs généraux pour les feuilletages obtenus par suspension que nous illustrerons par des exemples explicites.

Soit $\mathcal{U}=\left\{U_{i}\right\}$ un recouvrement ouvert de $M$. Pour tout multi-indice $\left(i_{0}, \cdots, i_{s}\right)$ tel que $U_{i_{0} \cdots i_{s}} \neq \emptyset, A^{k \ell}\left(U_{i_{0} \cdots i_{s}}\right)$ et $A^{q}\left(U_{i_{0} \cdots i_{s}}\right)$ seront les espaces 
de sections, au-dessus de $U_{i_{0} \cdots i_{s}}$, respectivement des faisceaux $\mathcal{A}^{k \ell}$ et $\mathcal{A}^{q}$. On pose

$$
C^{s}\left(\mathcal{U}, \mathcal{A}^{q}\right)=\prod_{\left(i_{o}, \cdots, i_{s}\right)} A^{q}\left(U_{i_{0} \cdots i_{s}}\right) .
$$

Un élément de $C^{s}\left(\mathcal{U}, \mathcal{A}^{q}\right)$ est appelé s-cochaîne sur $\mathcal{U}$ à valeurs dans $\mathcal{A}^{q}$; c'est une collection $\left\{\omega_{i_{0} \cdots i_{s}}\right\}$ où chaque composante $\omega_{i_{0} \cdots i_{s}}$ est un élément de $A^{q}\left(U_{i_{0} \cdots i_{s}}\right)$. On définit un opérateur $\delta_{s}: C^{s}\left(\mathcal{U}, \mathcal{A}^{q}\right) \longrightarrow C^{s+1}\left(\mathcal{U}, \mathcal{A}^{q}\right)$ de la façon suivante : pour toute $s$-cochaîne $c=\left\{\omega_{i_{0} \cdots i_{s}}\right\}, \delta_{s}(c)=\left\{\omega_{i_{0} \cdots i_{s+1}}\right\}$ sera la $(s+1)$-cochaîne telle que

$$
\omega_{i_{0} \cdots i_{s+1}}=\sum_{t=0}^{s+1}(-1)^{t} \omega_{i_{0} \cdots \widehat{i_{t}} \cdots i_{s+1}}
$$

où le signe $\hat{i}$ signifie qu'on a omis l'indice $i$. On vérifie aisément que, pour chaque $s, \delta_{s}$ est linéaire et qu'on a $\delta_{s+1} \circ \delta_{s}=0$. On pose d'autre part $D c=\left\{D \omega_{i_{o} \cdots i_{s}}\right\}$ et $\mathcal{D}=\delta_{s}+(-1)^{s} D$. On définit ainsi un opérateur

$$
\mathcal{D}: C^{s}\left(\mathcal{U}, \mathcal{A}^{q}\right) \longrightarrow C^{s+1}\left(\mathcal{U}, \mathcal{A}^{q}\right) \oplus C^{s}\left(\mathcal{U}, \mathcal{A}^{q+1}\right)
$$

dont il est facile de voir qu'il vérifie $\mathcal{D}^{2}=0$. On obtient donc un double complexe $\left(K^{s, q}, \mathcal{D}\right)$ avec

$$
K^{s, q}=C^{s}\left(\mathcal{U}, \mathcal{A}^{q}\right) \quad \text { et } \quad \mathcal{D}=\delta_{s}+(-1)^{s} D
$$

auquel est associée une suite spectrale de terme

$$
K_{2}^{s, q}=H^{s}\left(\mathcal{U}, \mathcal{H}^{q}\right)
$$

et convergeant vers $H^{*}\left(A^{*}(M)\right)=H^{*}\left(M, \mathcal{O}_{\mathcal{F}}\right)$; ici $\mathcal{H}^{q}$ désigne le préfaisceau qui à tout ouvert $U \in \mathcal{U}$ associe $H^{q}\left(U, \mathcal{O}_{\mathcal{F}}\right)$.

Supposons le recouvrement $\mathcal{U}$ acyclique ; alors $\mathcal{H}^{q}=0$ pour $q \geq 1$, la suite spectrale converge au terme $K_{2}$ et on a

$$
H^{s}\left(M, \mathcal{O}_{\mathcal{F}}\right)=H^{s}\left(\mathcal{U}, \mathcal{O}_{\mathcal{F}}\right) \text { pour tout } s \geq 0
$$

\section{Les revêtements feuilletés}

On considère l'exemple 1.2 (4) d'un revêtement feuilleté. On se donne un feuilletage transversalement holomorphe $\widetilde{\mathcal{F}}$ de codimension $n$ sur une variété $\widetilde{M}$ (de dimension $m+2 n$ ) et $\Gamma$ un groupe dénombrable opérant 
librement et proprement sur $\widetilde{M}$ par automorphismes de $\widetilde{\mathcal{F}}$ (en tant que feuilletage transversalement holomorphe). Alors la variété quotient $M=$ $\widetilde{M} / \Gamma$ est munie du feuilletage induit $\mathcal{F}$ qui est transversalement holomorphe de codimension $n$. Notons $\pi: \widetilde{M} \longrightarrow M$ la projection de revêtement ; c'est un morphisme de $(\widetilde{M}, \widetilde{\mathcal{F}})$ sur $(M, \mathcal{F})$; alors l'image réciproque $\pi^{*}\left(\mathcal{O}_{\mathcal{F}}\right) \mathrm{du}$ faisceau $\mathcal{O}_{\mathcal{F}}$ n'est rien d'autre que le faisceau $\mathcal{O}_{\tilde{\mathcal{F}}}$. Appliquant les résultats de $[\mathrm{Gr}]$ sur les foncteurs dérivés au revêtement $\pi: \widetilde{M} \longrightarrow M$ on obtient le

4.1. Théorème. Il existe une suite spectrale $E_{r}$ de terme $E_{2}^{k \ell}=H^{k}\left(\Gamma, H^{\ell}\left(\widetilde{M}, \mathcal{O}_{\tilde{\mathcal{F}}}\right)\right)$ et convergeant vers $H^{*}\left(M, \mathcal{O}_{\mathcal{F}}\right)$. (Les espaces vectoriels $H^{\ell}\left(\widetilde{M}, \mathcal{O}_{\tilde{\mathcal{F}}}\right)$ sont vus comme des $\Gamma$-modules. $)$

Si $\widetilde{M}$ est acyclique i.e.

$$
H^{\ell}\left(\widetilde{M}, \mathcal{O}_{\tilde{\mathcal{F}}}\right)=\left\{\begin{array}{ccc}
0 & \text { si } & l \geq 1 \\
\mathcal{O}_{\tilde{\mathcal{F}}}(\widetilde{M}) & \text { si } & l=0
\end{array}\right.
$$

la suite $E_{r}$ converge au terme $E_{2}$ et on a $H^{k}\left(M, \mathcal{O}_{\mathcal{F}}\right)=H^{k}\left(\Gamma, \mathcal{O}_{\tilde{\mathcal{F}}}(\widetilde{M})\right)$ où l'action de $\Gamma \operatorname{sur} \mathcal{O}_{\tilde{\mathcal{F}}}(\widetilde{M})$ est donnée par $(\gamma, f) \in \Gamma \times \mathcal{O}_{\tilde{\mathcal{F}}}(\widetilde{M}) \longmapsto f \circ \gamma^{-1} \in$ $\mathcal{O}_{\tilde{\mathcal{F}}}(\widetilde{M})$.

\subsection{L'exemple 3.2}

ii) On a $\widetilde{M}=\mathbf{C} \times \mathbf{R}-\{(0,0)\}$ munie du feuilletage transversalement holomorphe $\widetilde{\mathcal{F}}$ défini par $d z=0$ et $\Gamma=\mathbf{Z}$ agissant sur $\widetilde{M}$ à l'aide de la contraction réelle de rapport $\lambda \in] 0,1[$. On avait alors montré que $H^{1}\left(\widetilde{M}, \mathcal{O}_{\tilde{\mathcal{F}}}\right)=0$; le même type de calcul permet de montrer qu'en fait $H^{k}\left(\widetilde{M}, \mathcal{O}_{\tilde{\mathcal{F}}}\right)=0$ pour tout $k \geq 1$ i.e. $\widetilde{M}$ est acyclique. D'après 4.1 , on a

$$
H^{1}\left(M, \mathcal{O}_{\mathcal{F}}\right)=H^{1}\left(\mathbf{Z}, \mathcal{O}_{\tilde{\mathcal{F}}}(\widetilde{M})\right) .
$$

Il est facile de voir que $\left.\mathcal{O}_{\tilde{\mathcal{F}}}(\widetilde{M})\right)=\mathcal{O}(\mathbf{C})$; l'action de $\mathbf{Z}$ sur $\mathcal{O}(\mathbf{C})$ est celle qui à $f \in \mathcal{O}(\mathbf{C})$ associe la fonction holomorphe $z \in \mathbf{C} \longmapsto f(\lambda z) \in \mathbf{C}$. D'après le théorème $6.2, H^{1}\left(M, \mathcal{O}_{\mathcal{F}}\right)$ est isomorphe à $\mathbf{C}$.

4.3. Une remarque Mettons-nous dans la situation où $\widetilde{M}$ est une variété complexe, $\mathcal{F}$ le feuilletage par points et $\Gamma$ un groupe dénombrable agissant par biholomorphismes de façon libre et propre sur $\widetilde{M}$. Par le théorème 4.1, on a alors une suite spectrale de terme

$$
E_{2}^{k \ell}=H^{k}\left(\Gamma, H^{\ell}(\widetilde{M}, \widetilde{\mathcal{O}})\right)
$$

et convergeant vers $H^{*}(M, \mathcal{O})$ (où $\widetilde{\mathcal{O}}$ et $\mathcal{O}$ sont les faisceaux des germes de fonctions holomorphes respectivement sur $\widetilde{M}$ et $M$ ). Si les variétés $\widetilde{M}$ et $M$ 
sont 1-acycliques, on voit immédiatement que $H^{1}(\Gamma, \mathcal{O}(\widetilde{M}))=0$. Prenons en particulier $\Gamma=\mathbf{Z}$ et notons $\gamma$ le générateur de l'action sur $\widetilde{M}$; alors $H^{1}(\Gamma, \mathcal{O})$ est le quotient de $\mathcal{O}(\widetilde{M})$ par le sous-espace

$$
\mathcal{B}=\{f-f \circ \gamma: f \in \mathcal{O}(\widetilde{M})\} .
$$

Donc toute fonction holomorphe $g$ sur $\widetilde{M}$ s'écrit sous la forme $f-f \circ \gamma$ avec $f$ holomorphe sur $\widetilde{M}$. Le cas particulier de $\widetilde{M}=\mathbf{C}$ et $\gamma(z)=z+1$ a été résolu en 1887 par G. Guichard dans [Gu] (voir aussi [Wh]).

\section{Les suspensions}

On considère l'exemple $1.2(3)$; c'est un feuilletage transverse à la fibration $F \hookrightarrow M \stackrel{\pi}{\longrightarrow} B$. L'action, par biholomorphismes, de $\Gamma=\pi_{1}(B)$ sur $F$ induit une action sur la cohomologie de Dolbeault $H^{*}(F, \mathcal{O})(\mathcal{O}$ est le faisceau des germes de fonctions holomorphes sur $F$ ).

\section{1. $\Gamma$ est fini}

Si $\Gamma$ est fini (ou si la représentation $\Gamma \longrightarrow \operatorname{Aut}(F)$ se factorise à travers un quotient fini de $\Gamma$ ), alors le procédé de moyennisation

$$
\alpha \in A^{1}(\widetilde{M}) \longmapsto \frac{1}{\#(\Gamma)} \sum_{\gamma \in \Gamma} \gamma^{*}(\alpha) \in A_{\Gamma}^{1}(\widetilde{M})
$$

$\left(A_{\Gamma}^{1}(\widetilde{M})\right.$ étant l'espace des éléments $\Gamma$-invariants de $\left.A^{1}(\widetilde{M})\right)$ induit une injection

$$
H^{1}\left(M, \mathcal{O}_{\mathcal{F}}\right)=H^{1}\left(A_{\Gamma}^{*}(\widetilde{M})\right) \longrightarrow H^{1}\left(A^{*}(\widetilde{M})\right) .
$$

Donc $H^{1}\left(M, \mathcal{O}_{\mathcal{F}}\right)=0$ dès lors que $H^{1}\left(A^{*}(\widetilde{M})\right)$ est nul.

Désormais, nous supposerons que $\Gamma$ est infini et que la représentation $\Gamma \longrightarrow \operatorname{Aut}(F)$ ne se factorise pas à travers un groupe fini.

Pour tout $\ell=0, \cdots, n$, on a un fibré plat $\mathcal{H}^{\ell}$ sur $B$ de fibre $H^{\ell}(F, \mathcal{O})$ (en général de dimension infinie). Soit $\left\{W_{i}\right\}$ un bon recouvrement de $B$ i.e. toute intersection finie $U_{i_{0}} \cap \cdots \cap U_{i_{s}}$ est contractile (un tel recouvrement existe toujours ; il suffit de munir $B$ d'une métrique riemannienne et de la recouvrir par des boules ouvertes géodésiquement convexes). Pour chaque $i$ on pose $U_{i}=\pi^{-1}\left(W_{i}\right)$. On a alors $H^{*}\left(U_{i}, \mathcal{O}_{\mathcal{F}}\right)=H^{*}(F, \mathcal{O})$. En raisonnant comme dans $[\mathrm{BT}]$, on construit une suite spectrale de terme

$$
E_{2}^{k \ell}=H^{k}\left(B, \mathcal{H}^{\ell}\right)
$$

et convergeant vers $H^{*}\left(M, \mathcal{O}_{\mathcal{F}}\right)$. On sait alors que $H^{1}\left(M, \mathcal{O}_{\mathcal{F}}\right)=E_{\infty}^{10} \oplus E_{\infty}^{01}$. Mais comme $E_{\infty}^{10}=E_{2}^{10}=H^{1}(B, \mathcal{O})$, on obtient 


$$
H^{1}\left(M, \mathcal{O}_{\mathcal{F}}\right)=H^{1}(B, \mathcal{O}(F)) \oplus E_{\infty}^{01} .
$$

Ici $H^{k}(B, \mathcal{O}(F))$ est la cohomologie de de Rham de $B$ à valeurs dans le fibré plat sur $B$ de fibre l'espace de Fréchet $\mathcal{O}(F)$ et de monodromie définie par

$$
(\gamma, f) \in \Gamma \times \mathcal{O}(F) \longmapsto f \circ \gamma^{-1} \in \mathcal{O}(F) .
$$

\section{2. $F$ est 1 -acyclique}

Ceci est le cas si, par exemple, $F$ est une variété de Stein ( $c f$. [Hö] ou $[\mathrm{Ch}]$ pour la définition et les principales propriétés) ou une variété kählérienne compacte à premier nombre de Betti nul (cf. [We]). On a alors

$$
H^{1}\left(M, \mathcal{O}_{\mathcal{F}}\right)=H^{1}(B, \mathcal{O}(F))
$$

Si, en plus, $B$ est un espace classifiant i.e. $\pi_{i}(B)=0$ pour $i \geq 2$ (c'est le cas si le revêtement universel $\widetilde{B}$ est contractile) alors

$$
H^{1}\left(M, \mathcal{O}_{\mathcal{F}}\right)=H^{1}(B, \mathcal{O}(F))=H^{1}(\Gamma, \mathcal{O}(F))
$$

où $H^{\ell}(\Gamma, \mathcal{O}(F))$ est la cohomologie du groupe discret $\Gamma$ à valeurs dans le $\Gamma$-module $\mathcal{O}(F)$.

Supposons l'action de $\Gamma$ sur $F$ triviale ; dans ce cas $M$ est le produit $F \times B$ et les feuilles de $\mathcal{F}$ sont les fibres de la première projection $F \times B \longrightarrow$ $F$; en plus l'action de $\Gamma$ sur $\mathcal{O}(F)$ est aussi triviale de sorte que

$$
H^{1}\left(M, \mathcal{O}_{\mathcal{F}}\right)=H^{1}(B, \mathbf{C}) \otimes \mathcal{O}(F)=H^{1}(\Gamma, \mathbf{C}) \otimes \mathcal{O}(F) .
$$

Cette constatation nous sera très utile dans la démonstration du théorème qui suit dans lequel il n'est pas supposé que le feuilletage est une suspension.

5.3. Théorème. Soit $\mathcal{F}$ un feuilletage hermitien (i.e. riemannien et transversalement holomorphe) à feuilles fermées et chacune à groupe fondamental fini. Supposons en plus que toutes les feuilles ont le même revêtement universel L. Alors le problème additif de Cousin basique est équivalent au problème de Cousin sur l'orbifold complexe $W=M / \mathcal{F}$.

DÉmonstration. Remarquons d'abord que, si $M$ est compacte, d'après [Re], l'hypothèse de revêtement universel commun $L$ des feuilles est automatiquement satisfaite. 
Soient $y \in M$ et $L_{y}$ la feuille passant par $y$ qui est le quotient de $L$ par un groupe fini $\Gamma_{y}$. Alors $L_{y}$ admet un voisinage saturé $U$ qu'on peut décrire comme suit :

i) il existe un polydisque ouvert $V$ de $\mathbf{C}^{n}$ et une action isométrique biholomorphe de $\Gamma_{y}$ sur $V$;

ii) les fibres de la première projection $V \times L \longrightarrow V$ induisent un feuilletage $\mathcal{F}_{y}$ sur le quotient $(V \times L) / \Gamma_{y}$ (sur $L, \Gamma_{y}$ agit par automorphismes de revêtement) ;

iii) les variétés feuilletées $(U, \mathcal{F})$ et $\left((V \times L) / \Gamma_{y}, \mathcal{F}_{y}\right)$ sont isomorphes (en tant que variétés munies respectivement de feuilletages transversalement holomorphes).

D'autre part, on peut remarquer que :

- l'espace des feuilles de $\left((V \times L) / \Gamma_{y}, \mathcal{F}_{y}\right)$ s'identifie à $\bar{V}=V / \Gamma_{y}$,

- la variété $V$ a une cohomologie de Dolbeault triviale i.e. $H^{*}(V, \mathcal{O})=0$ pour $* \geq 1$ et $H^{0}(V, \mathcal{O})=\mathcal{O}(V)$. Par suite, le groupe $\Gamma_{y}$ étant fini, $\bar{V}$ a aussi une cohomologie de Dolbeault triviale,

- comme $\Gamma_{y}$ est fini, $H^{*}\left(U, \mathcal{O}_{\mathcal{F}}\right)$ s'injecte dans $H^{*}\left(V \times L / \Gamma_{y}, \mathcal{O}_{\mathcal{F}_{y}}\right)$.

Soit donc $\mathcal{U}=\left\{U_{i}\right\}$ un recouvrement de $M$ par des ouverts $U_{i}$ du type qu'on vient juste de décrire ; ce recouvrement se projette en un recouvrement ouvert $\overline{\mathcal{V}}=\left\{\bar{V}_{i}\right\}$ sur l'espace des feuilles $W=M / \mathcal{F}$. Les recouvrements $\mathcal{U}$ et $\overline{\mathcal{V}}$ définissent le même complexe simplicial. D'après 3.5 on a une suite spectrale

$$
K_{2}^{s q}=H^{s}\left(\mathcal{U}, \mathcal{H}^{q}\right)
$$

convergeant vers $H^{*}\left(M, \mathcal{O}_{\mathcal{F}}\right)$ où, comme toujours, $\mathcal{H}^{q}$ désigne le préfaisceau qui à tout ouvert $U \in \mathcal{U}$ associe $H^{q}\left(U, \mathcal{O}_{\mathcal{F}}\right)$. Mais le revêtement universel $\widetilde{U}_{i}$ de chaque ouvert $U \in \mathcal{U}$ est un produit $V \times L$ avec $V$ acyclique ; donc

$$
H^{q}\left(U, \mathcal{O}_{\mathcal{F}}\right)=H^{q}(L, \mathbf{C}) \otimes \mathcal{O}(\bar{V})
$$

Pour $q=0, H^{0}\left(U, \mathcal{O}_{\mathcal{F}}\right)$ n'est rien d'autre que l'espace $\mathcal{O}(\bar{V})$ des fonctions holomorphes sur $\bar{V}$. Par suite, tenant compte du fait que $H^{1}(L, \mathbf{C})=0$, on obtient

$$
H^{1}\left(M, \mathcal{O}_{\mathcal{F}}\right)=K_{\infty}^{10}=K_{2}^{10}=H^{1}(\overline{\mathcal{V}}, \mathcal{O})=H^{1}(W, \mathcal{O})
$$

Cette égalité montre bien que le feuilletage $\mathcal{F}$ est de Cousin si, et seulement si, l'orbifold $W$ est de Cousin. 


\section{Cas où $\Gamma=Z$}

Soient $F$ une variété complexe 1-acyclique et $\gamma: F \longrightarrow F$ un biholomorphisme. On considère la représentation $\Gamma=\pi_{1}\left(\mathbf{S}^{1}\right)=\mathbf{Z} \longrightarrow \operatorname{Aut}(F)$ qui au générateur 1 de $\mathbf{Z}$ associe le biholomorphisme $\gamma$. La suspension de cette représentation donne un feuilletage $\mathcal{F}$ transversalement holomorphe de dimension 1 sur la variété quotient

$$
M=(F \times \mathbf{R}) /(z, x) \simeq(\gamma(z), x+1) .
$$

\subsection{Description explicite de $H^{1}\left(M, \mathcal{O}_{\mathcal{F}}\right)$}

L'espace $H^{1}\left(M, \mathcal{O}_{\mathcal{F}}\right)$ est canoniquement isomorphe au quotient de $\mathcal{O}(F)$ par le sous-espace $\mathcal{B}=\{f-f \circ \gamma: f \in \mathcal{O}(F)\}$. La détermination de $\mathcal{B}$ se ramène donc à la résolution du problème suivant. Etant donnée une fonction $g \in \mathcal{O}(F)$, existe-t-il une fonction $f \in \mathcal{O}(F)$ solution de équation homologique

$$
f(z)-f(\gamma z)=g(z) ?
$$

Ce problème est souvent non trivial. Remarquons d'abord que, si $\gamma$ a un point fixe $z_{0}$, alors une condition nécessaire pour que $f$ existe est que $g\left(z_{0}\right)=0$. Si $\Sigma$ est l'ensemble des points fixes de $\gamma$, on notera $\mathcal{O}_{\Sigma}(F)$ l'espace des fonctions holomorphes sur $F$ qui s'annulent sur $\Sigma$; c'est un sous-espace fermé de $\mathcal{O}(F)$.

Soit $g \in \mathcal{O}_{\Sigma}(F)$; alors une solution formelle de l'équation (E) est donnée par la série suivante

$$
f(z)=\sum_{n=0}^{\infty} g\left(\gamma^{n} z\right)
$$

où $\gamma^{n}$ est le composé $n$-fois du biholomorphisme $\gamma$. Cette série définira une vraie solution si elle converge uniformément sur tout compact de $F$. Nous allons voir que ceci sera le cas pour certains biholomorphismes $\gamma$.

On munit $F$ d'une métrique riemannienne ; la norme induite sur chaque espace tangent sera notée \|\| . Si $\varphi: F \longrightarrow F$ est une application différentiable, ||$\left|\varphi^{\prime}(z)\right|||$ sera la norme de son application linéaire tangente $\varphi^{\prime}(z)$ au point $z \in F$ i.e.

$$
\|\| \varphi^{\prime}(z)\|\|=\sup _{\|u\| \leq 1}\left\|\varphi^{\prime}(z)(u)\right\| .
$$


Lorsque $z$ est un point fixe de $\varphi, \rho\left(\varphi^{\prime}(z)\right)$ sera son rayon spectral i.e. le nombre $\rho\left(\varphi^{\prime}(z)\right)=\lim \left(\left|\|\left(\varphi^{\prime}(z)\right)^{n}\right|||\right)^{\frac{1}{n}}$ qui est aussi $\max \{|\lambda|\}$ avec $\lambda$ valeur propre de $\varphi^{\prime}(z)$. Ce nombre ne dépend pas de la métrique choisie puisque sur un voisinage compact du point fixe toutes les métriques riemanniennes sont quasi-isométriques (deux métriques $h_{1}$ et $h_{2}$ sont quasi-isométriques s'il existe une constante $k \geq 1$ telle que $\frac{1}{k} h_{1} \leq h_{2} \leq k h_{1}$ ).

On suppose que $\gamma$ admet un seul point fixe 0 ; l'application linéaire tangente $\gamma^{\prime}(0)$ est un automorphisme de $T_{0} M$. Si $\rho\left(\gamma^{\prime}(0)\right)<1$ ou $\rho\left(\left(\gamma^{-1}\right)^{\prime}(0)\right)<$ 1 , on dira que 0 est un point hyperbolique

(i) Si $\rho\left(\varphi^{\prime}(0)\right)<1$ alors, pour tout $z \in F$, la suite $\gamma^{n}(z)$ tend vers 0 ; on dira que 0 est hyperbolique attractif.

(ii) Si $\rho\left(\left(\varphi^{-1}\right)^{\prime}(0)\right)<1$ alors, pour tout $z \in F$, la suite $\gamma^{-n}(z)$ tend vers 0 ; on dira que 0 est un point hyperbolique répulsif.

6.2. Théorème. Supposons que 0 est un point fixe hyperbolique attractif (resp. répulsif) pour $\gamma$. Soit $g$ une fonction holomorphe sur $F$ s'annulant en 0 ; alors la série $\sum_{n=0}^{\infty} g\left(\gamma^{n} z\right)$ (resp. la série $-\sum_{n<0} g\left(\gamma^{n} z\right)$ ) converge uniformément sur tout compact et définit sur $F$ une fonction holomorphe $f$ solution de l'équation $(E)$. Par conséquent $H^{1}\left(M, \mathcal{O}_{\mathcal{F}}\right) \simeq \mathbf{C}$.

DÉmonstration. On la fera dans le cas où 0 est attractif; le cas répulsif se traite de la même manière.

La série en question converge trivialement au point 0 puisque tous ses termes sont nuls. Pour montrer qu'elle converge uniformément sur un compact $K$, il suffit de montrer que la série des dérivées premières converge uniformément sur $K$. Soient $K$ un compact de $F$ et $z \in K$. On a

$$
\left(g \circ \gamma^{n}\right)^{\prime}(z)=g^{\prime}\left(\gamma^{n} x\right) \cdot \prod_{k=0}^{n-1} \gamma^{\prime}\left(\gamma^{k} z\right)
$$

où les produits sont les compositions des applications linéaires. Comme on a $\rho\left(\gamma^{\prime}(0)\right)<1$, il existe un réel $0<\rho<1$, une constante positive $C^{\prime}$ et un entier naturel $n_{0} \in \mathbf{N}$ tels que, pour $n$ suffisamment gand

$$
\left\|\prod_{k=0}^{n-1} \gamma^{\prime}\left(\gamma^{k} z\right)\right\| \| \text { est équivalent à } C^{\prime} \rho^{n-n_{0}} .
$$

D'autre part, comme $g^{\prime}$ est continue, il existe une constante positive $C^{\prime \prime}$ dépendant uniquement de $K$ et de $g$ telle que $\left\|g^{\prime}\left(\gamma^{n} z\right)\right\| \| \leq C^{\prime \prime}$ sur $K$. Ce qui donne

$$
\left\|\left|\left(g \circ \gamma^{n}\right)^{\prime}(z) \|\right| \leq C \rho^{n}\right.
$$


où $C$ est une constante positive ne dépendant que de $K, g$ et $n_{0}$. Comme $\rho<1$, la série

$$
g^{\prime}(z)+\sum_{n \geq 1} g^{\prime}\left(\gamma^{n} x\right) \cdot \prod_{k=0}^{n-1} \gamma^{\prime}\left(\gamma^{k} z\right)
$$

converge uniformément sur $K$; ce qui implique que la série $\sum_{n=0}^{\infty} g\left(\gamma^{n} x\right)$ converge uniformément sur $K$; celle-ci définit donc une fonction holomorphe $f$ sur $F$ solution de l'équation (E).

Définissons l'application $\mathcal{I}: \mathcal{O}(F) \longrightarrow \mathbf{C}$ par $\mathcal{I}(g)=g(0)$. C'est une forme linéaire continue sur l'espace $\mathcal{O}(F)$ non nulle (car $\mathcal{O}(F)$ contient les constantes) et son noyau est exactement l'espace des fonctions holomorphes sur $F$ qui s'écrivent $f-f \circ \gamma=g$ avec $g \in \mathcal{O}(F)$; d'où $H^{1}\left(M, \mathcal{O}_{\mathcal{F}}\right)=\mathbf{C}$. $\diamond$

\section{Suspension d'un automorphisme linéaire de $C^{n}$}

La richesse des calculs et les diverses propriétés qui vont intervenir ont fait mériter à cet exemple une section à part.

Soit $\gamma: \mathbf{C}^{n} \longrightarrow \mathbf{C}^{n}$ un automorphisme linéaire ayant toutes ses puissances différentes de l'identité. C'est un biholomorphisme de $F=\mathbf{C}^{n}$ dont la suspension donne un feuilletage transversalement holomorphe sur la variété $M=\mathbf{C}^{n} \times \mathbf{S}^{1}$. Le calcul de $H^{1}\left(M, \mathcal{O}_{\mathcal{F}}\right)$ se ramène donc toujours à la résolution de l'équation homologique $(\mathrm{E})$.

Le point 0 est fixé par $\gamma$. Soient $\lambda_{1}, \cdots, \lambda_{n}$ les valeurs propres de $\gamma$ et posons

$$
\alpha=\max \left\{\left|\lambda_{j}\right|: j=1, \cdots, n\right\} \text { et } \beta=\min \left\{\left|\lambda_{j}\right|: j=1, \cdots, n\right\} .
$$

Alors 0 est hyperbolique si, et seulement si, $\alpha<1$ ou $\beta>1$; dans ce cas 0 est le seul point fixe et les résultats de la section qui précède nous donnent $H^{1}\left(M, \mathcal{O}_{\mathcal{F}}\right)=\mathbf{C}$.

Lorsque 0 n'est pas hyperbolique (ce que l'on supposera désormais), le théorème 6.2 ne s'applique plus et le calcul peut être compliqué ; nous allons voir ce qu'il en est exactement.

\subsection{La dimension $n=1$}

(1) - L'automorphisme $\gamma$ s'écrit alors $\gamma(z)=a z$ où $a=|a| e^{2 i \pi \theta}$ (avec $\theta \in[0,1[)$ est un nombre complexe non nul. Comme on a supposé que le point fixe 0 est non hyperbolique on a en fait $|a|=1$; d'autre part, la représentation $1 \in \mathbf{Z} \longmapsto \gamma \in \mathrm{GL}(1, \mathbf{C})$ étant injective, $\theta$ est irrationnel. 
Pour résoudre l'équation homologique (E), on développe les fonctions $f$ et $g$ en séries entières

$$
f(z)=\sum_{n=0}^{\infty} b_{n} z^{n} \text { et } g(z)=\sum_{n=0}^{\infty} c_{n} z^{n} .
$$

Les coefficients $b_{n}$ et $c_{n}$ des deux séries sont des nombres complexes tels que, pour tout réel $R \geq 0$, les séries numériques

$$
\sum_{n=0}^{\infty}\left|b_{n}\right| R^{n} \text { et } \sum_{n=0}^{\infty}\left|c_{n}\right| R^{n} \text { convergent. }
$$

Ces conditions sont suffisantes pour assurer l'holomorphie de $f$ et $g$ sur $\mathbf{C}$. En les remplaçant par leurs développements respectifs en série, l'équation (E) devient équivalente au système

$$
\left(1-a^{n}\right) b_{n}=c_{n}, \quad n \in \mathbf{N} .
$$

On a $a^{n}=1$ uniquement lorsque $n=0$; le fait que $g$ s'annule en 0 impose au coefficient $c_{0}$ d'être nul. On pose alors

$$
b_{n}=\left\{\begin{array}{cc}
0 & \text { si } n=0 \\
\frac{c_{n}}{1-a^{n}} & \text { sinon. }
\end{array}\right.
$$

Mais la collection $\left\{b_{n}\right\}$ ne définit pas toujours une série convergente $\sum b_{n} z^{n}$ car le sous-ensemble $\left\{a^{n}: n \in \mathbf{N}\right\}$ est dense dans le cercle et une sous-suite $\left(1-a^{n_{s}}\right)$ peut tendre vers 0 beaucoup plus vite que la suite $\left(c_{n}\right)$. Ce qui va faire intervenir la nature arithmétique du nombre $\theta$. Nous étudierons deux cas : $\theta$ diophantien et $\theta$ super-Liouville.

(i) - $\theta$ est diophantien

Cela signifie qu'il existe des nombres réels $A>0$ et $\delta \geq 2$ tels que

$$
\left|1-a^{n}\right| \geq \frac{A}{|n|^{\delta}}
$$

pour tout $n \in \mathbf{Z}^{*}$. Ceci nous donne l'inégalité $\left|b_{n}\right| \leq A n^{\delta}\left|c_{n}\right|$ pour $n \in \mathbf{N}^{*}$. La série entière $\sum b_{n} z^{n}$ converge donc uniformément sur tout compact de $\mathbf{C}$ et définit une fonction holomorphe $f \in \mathcal{O}(\mathbf{C})$ solution de l'équation homologique $(\mathrm{E})$. L'espace $H^{1}\left(M, \mathcal{O}_{\mathcal{F}}\right)$ est isomorphe à $\mathbf{C}$.

(ii) - $\theta$ est "super-Liouville" 
Par définition, il existe une constante $A>0$ et pour tout entier $s>1$, il existe un entier naturel $n_{s}$ tel que

$$
\left|1-e^{2 i \pi n_{s} \theta}\right| \leq A e^{-s}
$$

Cela signifie que le nombre 1 est "très bien approché" par les nombres complexes du type $e^{2 i \pi n_{s} \theta}$. On pose alors

$$
c_{n}=\left\{\begin{array}{cc}
0 & \text { pour } n \neq n_{s} \text { ou } n=0 \\
e^{-\frac{s}{2}} & \text { pour } n=n_{s}
\end{array}\right.
$$

Il est facile de voir que la série $\sum c_{n} z^{n}$ définit bien une fonction holomorphe $g$ sur $\mathbf{C}$ vérifiant $g(0)=0$ mais que la série $\sum b_{n} z^{n}$ ne converge pas ; en effet les coefficients $b_{n}$ vérifient l'inégalité $\left|b_{n_{s}}\right| \geq \frac{1}{A} e^{\frac{s}{2}}$ et ne tendent donc pas vers 0 . De cette façon on peut construire une famille infinie libre de fonctions $g_{\ell}$ holomorphes vérifiant $g_{\ell}(0)=0$ pour lesquelles l'équation homologique $(\mathrm{E})$ n'a pas de solution. Ainsi l'espace vectoriel $H^{1}\left(M, \mathcal{O}_{\mathcal{F}}\right)$ est de dimension infinie. On peut remarquer que, pour les polynômes $g$ à terme constant nul, il y a toujours une solution ; comme l'adhérence du sous-espace qu'ils engendrent est de codimension 1 , l'espace $H^{1}\left(M, \mathcal{O}_{\mathcal{F}}\right)$ n'est pas séparé, mais son séparé associé (i.e. le quotient de $\mathcal{O}(\mathbf{C})$ par l'adhérence du sous-espace $\{f-f \circ \gamma: f \in \mathcal{O}(\mathbf{C})\})$ est isomorphe à $\mathbf{C}$.

\subsection{La dimension supérieure}

Comme nous l'avons déjà dit, les calculs sont très compliqués. Nous nous contenterons de les faire pour $n=2$ qui est déjà une situation non triviale. On notera $a$ et $b$ les valeurs propres de $\gamma$ qui sont toutes les deux non nulles. Trois cas sont possibles :

(1) $a=b$ et $\gamma$ est diagonalisable ; $\gamma$ est alors conjugué à l'homothétie de rapport $a$,

(2) $a=b$ de module 1 et $\gamma$ n'est pas diagonalisable ; $\gamma$ est alors conjugué à l'automorphisme $(z, w) \longmapsto(a z+w, a w)$.

(3) $a \neq b$ et donc $\gamma$ est conjugué à l'automorphisme $(z, w) \longmapsto(a z, b w)$.

Il suffit donc de faire les calculs successivement pour les transformations linéaires suivantes

$$
(z, w) \stackrel{\gamma}{\longmapsto}(a z, a w), \quad(z, w) \stackrel{\gamma}{\longmapsto}(a z+w, a w) \text { et }(z, w) \stackrel{\gamma}{\longmapsto}(a z, b w)
$$

On peut remarquer que dans les cas (1) et (2) on peut écrire $a=e^{2 i \pi \theta}$ ; comme on a supposé la représentation $1 \in \mathbf{Z} \longmapsto \gamma \in \mathrm{GL}(2, \mathbf{C})$ injective, $\theta$ est forcément irrationnel. 
Toute fonction holomorphe $g: \mathbf{C}^{2} \longrightarrow \mathbf{C}$ se laisse développer sous forme de série entière

$$
g(z, w)=\sum_{m, n \in \mathbf{N}} c_{m n} z^{m} w^{n}
$$

où la suite double des coefficients $c_{m n}$ est telle que, pour tout réel $R \geq 0$, la série $\sum_{m, n}\left|c_{m n}\right| R^{m+n}$ converge. Etant donnée une telle fonction $g$, on cherche une fonction holomorphe $f$ sur $\mathbf{C}^{2}$ telle que $f-f \circ \gamma=g$. Comme on l'a déjà remarqué, une condition nécessaire d'existence de $f$ est $c_{00}=0$ que nous supposerons désormais remplie.

On se donne donc une fonction holomorphe $g$ sur $\mathbf{C}^{2}$ et on cherche une fonction holomorphe $f$ sur $\mathbf{C}^{2}$ telle que pour tout $(z, w) \in \mathbf{C}^{2}$ on ait

$$
f(z, w)-f(\gamma(z, w))=g(z, w) .
$$

Pour résoudre cette équation, on utilisera les développements en série entière des fonctions $f$ et $g$ :

$$
f(z, w)=\sum_{m, n=0}^{\infty} b_{m n} z^{m} w^{n} \text { et } g(z, w)=\sum_{m, n=0}^{\infty} c_{m n} z^{m} w^{n} .
$$

\section{$\underline{\operatorname{Cas}(1)}$}

L'automorphisme $\gamma$ étant une homothétie, ce cas se ramène à la dimension $n=1$. Nous renvoyons donc le lecteur à la sous-section 7.1.

$\underline{\text { Cas }(2)}$

Si $|a| \neq 1,0$ est un point fixe hyperbolique ; on peut donc appliquer le théorème 6.2 et montrer que $H^{1}\left(M, \mathcal{O}_{\mathcal{F}}\right)=\mathbf{C}$.

Le cas $|a|=1$ est loin d'être trivial et nous n'avons aucune méthode d'attaque ; nous nous contenterons de la réponse partielle qui suit et considérerons que, de façon générale, la question est ouverte. Toute solution de l'équation $f(z, w)-f(a z+w, a w)=g(z, w)$ donne une solution de $f(z, 0)-f(a z, 0)=g(z, 0)$; autrement dit, on récupère le problème en dimension 1 sur le sous-espace vectoriel d'équation $w=0$. Si donc $\theta$ est un nombre "super-Liouville", l'espace vectoriel $H^{1}\left(M, \mathcal{O}_{\mathcal{F}}\right)$ est de dimension infinie non séparé.

\section{$\underline{\text { Cas (3) }}$}

En remplaçant $f$ et $g$ par leurs développements respectifs en série entière, on obtient le système (au niveau des coefficients $c_{m n}$ et $b_{m n}$ )

$$
\left(1-a^{m} b^{n}\right) b_{m n}=c_{m n}, \quad m, n \in \mathbf{N} .
$$


(On rappelle qu'on a supposé $c_{00}=0$ qui vient de la condition nécessaire $g(0,0)=0$ d'existence d'une solution pour l'équation.) On pose $a=r e^{2 i \pi \theta}$ et $b=\rho e^{2 i \pi \sigma}(\operatorname{avec} \theta, \sigma \in[0,1[) ;$ soit

$$
E=\left\{(m, n) \in \mathbf{N}^{2}: a^{m} b^{n}=1\right\} .
$$

L'ensemble $E$ est non vide puisqu'il contient $(0,0)$ et est invariant par toute application $(m, n) \in \mathbf{N}^{2} \longmapsto(k m, k n) \in \mathbf{N}^{2}$ où $k \in \mathbf{N}$ i.e. si $E$ contient $(m, n)$, il contient tous ses multiples $k(m, n)$; d'autre part $(m, n) \in E$ si, et seulement si

$$
r^{m} \rho^{n}=1 \text { et } m \theta+n \sigma \text { est entier naturel. }
$$

Posons $u=\ln r, v=\ln \rho, \tau=\frac{u}{v}$ et $\eta=\frac{\theta}{\sigma}$. Alors la condition $r^{m} \rho^{n}=1$ est équivalente à $m u+n v=0$.

Trois sous-cas se présentent : i) $r=\rho=1$, ii) $r=1$ et $\rho \neq 1$ (ou $\rho=1$ et $r \neq 1$ ) et iii) $r<1$ et $\rho>1$ (ou $r>1$ et $\rho<1$ ).

\section{Sous-cas (i)}

L'égalité $r^{m} \rho^{n}=1$ est satisfaite pour tout $(m, n)$. Reste à examiner le nombre $m \theta+n \sigma$. Soit $L: \mathbf{Z}^{2} \longrightarrow \mathbf{R}$ l'application définie par $L(m, n)=$ $m \theta+n \sigma ; L$ est un morphisme de groupes. On pose $\mathcal{G}=L\left(\mathbf{N}^{2}\right) \cap \mathbf{N}$. On aura plusieurs possibilités.

La première : $\mathcal{G}=\{0\}$

Alors l'ensemble $E$ est réduit à $\{(0,0)\}$ et on peut calculer les coefficients $b_{m n}$ en posant

$$
b_{m n}=\left\{\begin{array}{cc}
0 & \text { si }(m, n)=(0,0) \\
\frac{c_{m, n}}{1-a^{m} b^{n}} & \text { sinon. }
\end{array}\right.
$$

D'autre part, comme la représentation $1 \in \mathbf{Z}=\Gamma \longmapsto \gamma \in \mathrm{GL}(2, \mathbf{C})$ est injective, le nombre 1 est dans l'adhérence de $\Sigma$ et on est alors confronté à la manière dont les éléments de $\Sigma$ approchent $1 \mathrm{ou}$, de façon équivalente, aux propriétés arithmétiques de $\eta=\frac{\theta}{\sigma}$ : si $\eta$ est diophantien, les coefficients $b_{m n}$ définissent une fonction holomorphe $f$ répondant à la question et donc $H^{1}\left(M, \mathcal{O}_{\mathcal{F}}\right)=\mathbf{C}$; si $\eta$ est "super-Liouville", on montre comme en dimension $n=1$ que $H^{1}\left(M, \mathcal{O}_{\mathcal{F}}\right)$ est de dimension infinie non séparé mais que son séparé associé est de dimension 1.

La deuxième $: \mathcal{G} \neq\{0\}$ 
Alors $\mathcal{G}$ est un sous-demi-groupe de type $d \mathbf{N}$ avec $d \in \mathbf{N}^{*}$. Soit $\Sigma=$ $L^{-1}(\mathcal{G}) \cap \mathbf{N} \times \times N$. C'est un sous-demi-groupe infini de $\mathbf{N} \times \mathbf{N}$. On pose

$$
b_{m n}=\left\{\begin{array}{cc}
0 & \text { si }(m, n) \in \sum \\
\frac{c_{m, n}}{1-a^{m} b^{n}} & \text { sinon. }
\end{array}\right.
$$

(*) Si $\eta$ est diophantien, les coefficients $b_{m n}$ définissent une fonction holomorphe $f$ répondant à la question ; par suite $H^{1}\left(M, \mathcal{O}_{\mathcal{F}}\right)$ est de dimension infinie séparé et engendré par $\left\{z^{m} w^{n}:(m, n) \in \Sigma\right\}$.

$(* *)$ Si $\eta$ est "super-Liouville", on montre, comme précédemment, que $H^{1}\left(M, \mathcal{O}_{\mathcal{F}}\right)$ est de dimension infinie non séparé mais que son séparé associé est engendré par $\left\{z^{m} w^{n}:(m, n) \in \Sigma\right\}$.

$\underline{\text { Sous-cas (ii) }}$

On ne peut avoir $r^{m} \rho^{n}=1$ que pour $n=0$ mais alors $m \theta+n \sigma=m \theta$ ; par suite, pour pouvoir résoudre il faut supposer $b_{m 0}=0$; ceci étant on pose

$$
b_{m n}=\left\{\begin{array}{cc}
0 & \text { si } n=0 \\
\frac{c_{m, n}}{1-a^{m} b^{n}} & \text { sinon. }
\end{array}\right.
$$

Première possibilité : $\theta$ rationnel

Ecrivons $\theta=\frac{p}{q}$ avec $p \in \mathbf{N}, q \in \mathbf{N}^{*}$ et $p$ et $q$ premiers entre eux. Posons

$$
\delta=\min \left\{\left|1-a^{\ell} b^{n}\right|\right\}
$$

où le minimum est pris sur $\ell=0, \cdots, q-1$ et $n \neq 0$. Le nombre $\delta$ est strictement positif et pour tout $(m, n)$ on a l'estimation

$$
\left|b_{m n}\right| \leq \frac{1}{\delta}\left|c_{m n}\right|
$$

Par suite les coefficients $b_{m n}$ définissent bien la fonction holomorphe cherchée $f$. Donc $H^{1}\left(M, \mathcal{O}_{\mathcal{F}}\right)$ est de dimension infinie séparé et engendré par les monômes $\left\{z^{\ell q} w^{n}: \ell, n \in \mathbf{N}\right\}$.

Deuxième possibilité : $\theta$ irrationnel

Si $\theta$ est diophantien, les coefficients $b_{m n}$ définissent une fonction holomorphe $f$ répondant à la question et donc $H^{1}\left(M, \mathcal{O}_{\mathcal{F}}\right)$ est de dimension infinie séparé engendré par les monômes $\left\{z^{m}: m \in \mathbf{N}\right\}$; si $\theta$ est "super-Liouville", on montre que $H^{1}\left(M, \mathcal{O}_{\mathcal{F}}\right)$ est de dimension infinie non séparé mais que son séparé associé est aussi engendré par les monômes $\left\{z^{m}: m \in \mathbf{N}\right\}$.

Sous-cas (iii) 
Un couple $\left(m_{0}, n_{0}\right)$ est dans $E$ si, et seulement si, $m_{0} u+n_{0} v=0$ et le nombre $m_{0} \theta+n_{0} \sigma$ est un entier. Alors (on suppose $m_{0} \neq 0$ ) le nombre $\tau=\frac{u}{v}$ est le rationnel $-\frac{n_{0}}{m_{0}}$. Tout autre couple $(m, n)$ vérifiant $m u+n v=0$ est de la forme $\left(k m_{0}, k n_{0}\right)$ avec $k \in \mathbf{N}$.

Première possibilité : $E=\{(0,0)\}$

On suppose alors que le coefficient $c_{00}$ est nul et on définit les coefficients $b_{m n}$ comme précédemment par les formules

$$
b_{m n}=\left\{\begin{array}{cc}
0 & \text { si }(m, n)=(0,0) \\
\frac{c_{m, n}}{1-a^{m} b^{n}} & \text { sinon. }
\end{array}\right.
$$

Comment 1 est-il approché par les nombres $a^{m} b^{n}$ ? C'est équivalent à la manière dont le nombre $\tau=\frac{u}{v}$ est approché par les rationnels : ceci découle du théorème des accroissements finis appliqué en 0 à la fonction exponentielle. Par conséquent si $\tau$ est diophantien, les coefficients $b_{m n}$ définissent une fonction holomorphe $f$ répondant à la question et donc $H^{1}\left(M, \mathcal{O}_{\mathcal{F}}\right)=\mathbf{C}$; si $\tau$ est "super-Liouville", on montre que $H^{1}\left(M, \mathcal{O}_{\mathcal{F}}\right)$ est de dimension infinie non séparé mais que son séparé associé est de codimension 1 .

Deuxième possibilité : $E=\left\{\left(k m_{0}, k n_{0}\right): k \in \mathbf{N},\left(m_{0}, n_{0}\right) \neq(0,0)\right\}$

On a donc à la fois $r^{m_{0}} \rho^{n_{0}}=1$ et $m_{0} \theta+n_{0} \sigma$ entier. On suppose alors que les coefficients $c_{m n}$ avec $(m, n)=\left(k m_{0}, k n_{0}\right)$ sont nuls et on définit les coefficients $b_{m n}$ comme précédemment par les formules

$$
b_{m n}=\left\{\begin{array}{cc}
0 & \text { si }(m, n)=\left(k m_{0}, k n_{0}\right) \\
\frac{c_{m, n}}{1-a^{m} b^{n}} & \text { sinon. }
\end{array}\right.
$$

Par le même type de raisonnement appliqué plusieurs fois jusqu'à présent on montre que : si $\xi=\frac{\sigma}{\theta}$ est diophantien, les coefficients $b_{m n}$ définissent une fonction holomorphe $f$ répondant à la question et donc $H^{1}\left(M, \mathcal{O}_{\mathcal{F}}\right)$ est de dimension infinie séparé engendré par les monômes $\left\{\left(z^{m_{0}} w^{n_{0}}\right)^{k}: k \in \mathbf{N}\right\}$ ; si $\xi$ est "super-Liouville", $H^{1}\left(M, \mathcal{O}_{\mathcal{F}}\right)$ est de dimension infinie non séparé mais que son séparé associé est engendré lui aussi par les monômes de la forme $\left(z^{m_{0}} w^{n_{0}}\right)^{k}$ avec $k$ variant dans $\mathbf{N}$.

Les calculs de groupes de cohomologie que nous venons de donner, ont utilisé de manière substantielle les propriétés arithmétiques des nombres irrationnels. La littérature est abondante à ce sujet et le lecteur désireux d'y plonger plus profondément peut consulter par exemple [CC], [La] ou [Sc]. 


\section{Un feuilletage de Cousin}

La question de savoir s'il existe des feuilletages de Cousin est naturellement importante. Nous avons vu (théorème 5.3) que ceci se produit pour un feuilletage riemannien dont les feuilles sont compactes et à groupe fondamental fini et tel que le quotient soit une orbifold complexe à premier groupe de cohomologie de Dolbeault nul. Nous allons en donner un ayant une dynamique un peu plus compliquée.

On note $\operatorname{SL}(n, \mathbf{R})$ le groupe des matrices réelles $n \times n$ de déterminant 1. C'est une forme réelle du groupe $\operatorname{SL}(n, \mathbf{C}$ ) (matrices $n \times n$ complexes de déterminant égal à 1) ; celui-ci agit par transformations projectives sur $P^{n-1}(\mathbf{C})$ (espace projectif complexe de dimension $\left.n-1\right)$. Donc tout sousgroupe de $\operatorname{SL}(n, \mathbf{C})$ agit de façon similaire sur $P^{n-1}(\mathbf{C})$.

La construction du groupe $\Gamma$ suivant et ses propriétés se trouvent dans [Mi]. Dans le demi-plan de Lobachevsky $\mathbf{H}=\{z=x+i y: y>0\}$ muni de la métrique de Poincaré $\frac{d x^{2}+d y^{2}}{y^{2}}$ on prend un triangle géodésique $T(p, q, r)$ d'angles $\frac{\pi}{p}, \frac{\pi}{q}$ et $\frac{\pi}{r}$ avec $\frac{1}{p}+\frac{1}{q}+\frac{1}{r}<1$. On note $\sigma_{1}, \sigma_{2}$ et $\sigma_{3}$ les réflexions respectives par rapport aux trois côtés ; celles-ci engendrent un groupe d'isométries $\Sigma^{*}$; les éléments qui préservent l'orientation forment un sousgroupe $\Sigma$ de $\Sigma^{*}$ d'indice 2 qu'on appelle le groupe du triangle $T(p, q, r)$. C'est un sous-groupe de $\mathrm{SL}(2, \mathbf{R})$ et son image réciproque $\Gamma$ par le morphisme projection $\widetilde{\mathrm{SL}}(2, \mathbf{R}) \longrightarrow \mathrm{SL}(2, \mathbf{R})(\widetilde{\mathrm{SL}}(2, \mathbf{R})$ étant le revêtement universel de $\mathrm{SL}(2, \mathbf{R}))$ est une extension centrale

$$
0 \longrightarrow \mathbf{Z} \longrightarrow \Gamma \longrightarrow \Sigma \longrightarrow 1 \text {. }
$$

Le groupe $\Gamma$ admet comme présentation

$$
\Gamma=\left\langle\gamma_{1}, \gamma_{2}, \gamma_{3} \mid \gamma_{1}^{p}=\gamma_{2}^{q}=\gamma_{3}^{r}=\gamma_{1} \gamma_{2} \gamma_{3}\right\rangle=1 .
$$

Le quotient $B=\widetilde{\mathrm{SL}}(2, \mathbf{R}) / \Gamma$ est une variété compacte de dimension 3 . Si les entiers $p, q$ et $r$ sont premiers entre eux deux à deux la cohomologie (à coefficients dans $\mathbf{Z}$ ) de $B$ est celle de la sphère $\mathbf{S}^{3}$; en particulier $H^{1}(B, \mathbf{C})=H^{1}\left(\mathbf{S}^{3}, \mathbf{C}\right)=0$. En tant que sous-groupe de $\widetilde{\mathrm{SL}}(2, \mathbf{R}), \Gamma$ agit sur l'espace projectif $P^{1}(\mathbf{C})$. On obtient donc une représentation (non injective car elle se factorise par le morphisme projection $\widetilde{\mathrm{SL}}(2, \mathbf{R}) \longrightarrow \mathrm{SL}(2, \mathbf{R}))$

$$
\rho: \pi_{1}(B)=\Gamma \longrightarrow \operatorname{Aut}\left(P^{1}(\mathbf{C})\right) .
$$

La suspension d'une telle représentation donne un feuilletage transversalement holomorphe $\mathcal{F}$ de codimension 1 sur la variété différentiable $M$ de dimension 5 , quotient de $\widetilde{M}=P^{1}(\mathbf{C}) \times \widetilde{\mathrm{SL}}(2, \mathbf{R})$ par la relation d'équivalence 
identifiant $(z, x)$ à $(\rho(\gamma)(z), \gamma x)$ avec $\gamma \in \Gamma(\Gamma$ agissant sur $\widetilde{\mathrm{SL}}(2, \mathbf{R})$ par translations à gauche). Les feuilles de $\mathcal{F}$ sont des espaces homogènes de $\widetilde{\mathrm{SL}}(2, \mathbf{R})$ par des sous-groupes discrets. On sait que

$$
H^{\ell}\left(P^{1}(\mathbf{C}), \mathcal{O}\right)=\left\{\begin{array}{cc}
C & \text { si } l=0 \\
0 & \text { si } l \geq 1 .
\end{array}\right.
$$

D'après 4.1 on a $H^{1}\left(M, \mathcal{O}_{\mathcal{F}}\right)=H^{1}(B, \mathbf{C})=0$; le feuilletage $(M, \mathcal{F})$ est donc de Cousin.

\section{Références}

[BT] Bотт, R. \& Tu, L. Differential Forms in Algebraic Topology. GTM $n^{\circ}$ 82, Springer-Verlag, (1982).

[Ch] Chabat, B. Introduction à l'analyse complexe, Tome 2 : Fonctions de plusieurs variables complexes. Editions MIR, Moscou (1990).

[CC] Cilleruelo, J. \& Cordoba, A. La teoría de los números. Biblioteca Mondadori, Madrid (1992).

[Co] Cousin, P. Sur les fonctions de $n$ variables complexes. Acta Mathematica 19, (1895).

[DK] Duchamp, T. \& Kalka, M. Deformation theory for holomorphic foliations. J. of Diff. Geom. 14, (1979) 317-337.

[Ek1] El Kacimi Alaoui, A. Dualité pour les feuilletages transversalement holomorphes. Manuscripta Math. 58, (1987) 417-443.

[Ek2] El Kacimi Alaoui, A. Opérateurs transversalement elliptiques sur un feuilletage riemannien et applications. Compositio Math. 73, (1990) $57-106$.

[Ek3] El Kacimi Alaoui, A. Sur le problème du $\bar{\partial}$ le long des feuilles. Prépublication (mars 2003).

[GT] Gigante, G. \& Tomassini, G. Foliations with complex leaves. Diff. Geo. and its Applications 5, (1995) 33-49.

[Gr] Grothendieck, A. Sur quelques points d'algèbre homologique. Tôhoku Math. J. Vol. 9, (1957) 119-221.

[Gu] Guichard, C. Sur la résolution de l'équation aux différences finies $G(x+1)-G(x)=H(x)$. Ann. Sc. ENS 4 (1887) 361-380.

[Hö] Hörmander, L. An Introduction to Complex Analysis in Several Variables. North-Holland, Vol. 7, (1990). 
[La] LANG, S. Report on Diophantine approximation. Bulletin de la SMF 93, (1965) 117-192.

[Mi] Milnor, J. On the 3-dimensional Brieskorn Manifolds $M(p, q, r)$. Ann. of Math. Studies Knots, Groups and 3-Manifolds edited by L. P. NeUWIRTH, (1975) 175-225.

[MK] Morrow, J. \& Kodaira, K. Complex Manifolds. Athena Series, Selected Topics in Mathematics, Holt, Rinehart and Winston, New York (1971).

[Re] Reinhart, B.L. Foliated manifolds with bundle-like metrics. Ann. of Math. 69, (1959) 119-132.

[Sc] Schmidt, W. M. Diophantine approximation. Lecture Notes in Math. 785, (1980).

[Va] Vaisman, I. Sur la cohomologie des variétés analytiques complexes feuilletées. C.R. Acad. Sc. Paris tome 273, (1971) 1067-1070.

[We] Wells, R. O. Differential Analysis on Complex Manifolds. Graduate Text in Math. 65, (1979).

[Wh] Whittaker, J. M. Interpolatory Function Theory. Cambridge Tracts in Math. and Physic (1964).

Received : January, 2003.

\author{
A. El Kacimi Alaoui \\ LAMATH, Le Mont Houy \\ Université de Valenciennes \\ 59313 Valenciennes Cedex 9 \\ France \\ e-mail : aziz.elkacimi@univ-valenciennes.fr

\section{T. Sohou} \\ Université de Cocody, UFR de Mathématiques \\ 22 BP 582 - Abidjan 22 - Côte d'Ivoire \\ France \\ e-mail : sohout@syfed.ci.refer.org
}

